\title{
Applications of Rough Soft to Extensions Semihypergroups Induced by Operators and Corresponding Decision Making Methods
}

\author{
N. Rakhsh Khorshid ${ }^{1}$, S. Ostadhadi-Dehkordi ${ }^{2}$ \\ ${ }^{1,2}$ Department of Mathematics, University of Hormozgan, Bandar Abbas, Iran. \\ n.rakhshkhorshid.phd@hormozgan.ac.ir \\ Ostadhadi@hormozgan.ac.ir
}

\begin{abstract}
In this article, we apply rough soft set to a special algebraic hyperstructure, which obtained of disjoint $\Gamma$-semihypergroups, and give the concept of rough soft semihypergroup. We propose the notion of lower and upper approximations with respect to a special semihypergroup and obtain some properties of them. Moreover, we consider a connection between lower(upper) approximation of a special semihypergroup and lower(upper) approximation of associated $\Gamma$-hypergroupoid.

In the last section of this research, we discuss the decision making algorithm of rough soft semihypergroups and we obtain a relation between the decision making algorithm of rough soft semihypergroups and their associated rough soft $\Gamma$-hypergroupoid for a special semihypergroup.

Key words and phrases: semihypergroup, $\Gamma$-semihypergroup, regular relation, Pawlak space, rough soft set, decision making.
\end{abstract}

\section{Introduction}

A semigroup is an algebraic structure consisting of a nonempty set equipped with an associative binary operation in the set. semigroup plays an important role in some areas of mathematics, among others, in coding theory, 
combinatories, and mathematical analysis. In 1986, Sen and Saha [43] defined an idea $\Gamma$-semigroup which was a generalization of a semigroup. Some classical ideas from semigroup have been developed in $\Gamma$-semigroups such as communitativity, regularity, and ideal $[46,23,4]$ and many others.

The concept of algebraic hyperstructure theory was introduced in 1934 by Marty [27]. The Hyperstructures theory have many applications in hypergraphs and graphs, binary relations, lattices, fuzzy sets and rough sets, automata, cryptography, codes, artificial intelligence and probabilities $[7,8,10]$. The concept of $\Gamma$-semihypergroup as a generalization of semigroup, semihypergroup and $\Gamma$-semigroup Davvaz et al. introduced by $[2,9,21,20,22]$.

The rough set theory, introduced by Pawlak $[39,40]$ and he used this concept as a method for data mining. Also, many researchers and practitioners in various field of science and technology work on this concept and there are many applications in various areas such as knowledge discovery, economics, finance, engineering and even arts and culture[25, 28, 31, 38, 41, 42, 44, 45, $47,48]$. There are many problems in the real life such as engineering, medical science, economic, environments, etc. have various uncertainties. Some kinds of theories were given like theory of fuzzy sets[48], theory of vague set[19] and rough sets[37]. The concept of soft set theory introduced by Molodsov[35] as a mathematical tool for handling uncertainties. He applied this theory to several directions [33, 34, 35]. The application of soft set in the decision making problem considered by Maji et.al. [29, 30]. Also, Ma et. al. [26] introduce and consider decision making algorithm of rough soft sets to Krasner $(m, n)$-hyperrings. The concept soft groups introduced and studied by Aktas et.al.[1]. Feng et al. [15] studied interesting connection between two mathematical approaches to vagueness: Rough sets and soft sets, called soft approximation spaces and soft rough sets.

This paper is structured as follows. We define a new set and we introduce the semihypergroup associated with this new hyperstructure that obtained of union of two $\Gamma$-semihypergroups which don't meet each other and we study relations between them. In the third section, we define rough semihypergroups, soft semihypergroups and prove that there is relation between them and associated rough and soft $\Gamma$-hypergroupoid. Also, we define rough soft semihypergroups by defining a regular relation and show that there are relations between rough soft semihypergroups and their associated rough soft $\Gamma$-hypergroupoid. Finally, in the last section, we present the decision making algorithm of rough soft semihypergroups, then we find a relation between the decision making algorithm of this rough soft semihypergroup and its associated rough soft $\Gamma$-hypergroupoid. 


\section{$2 G_{1}\left[G_{2}\right]$ and associated semihypergroup}

In this section, we introduce the semihypergroup associated with $G_{1}\left[G_{2}\right]$, where $G_{1}$ and $G_{2}$ are $\Gamma$-semihypergroups such that $G_{1} \cap G_{2}=\emptyset$ and study relations between them.

Definition 2.1. Let $H$ and $\Gamma$ be non-empty sets and for every $\alpha$ of $\Gamma$ there are hyperoperations $\otimes_{\alpha}: H \times H \longrightarrow P^{*}(H)$. Then, we say that $H$ is a $\Gamma$-semihypergroup, when

$$
\left(x \otimes_{\alpha} y\right) \otimes_{\beta} z=x \otimes_{\alpha}\left(y \otimes_{\beta} z\right),
$$

for every $\alpha$ and $\beta$ of $\Gamma$ and $x, y, z \in H$. A $\Gamma$-semihypergroup $H$ is called $\Gamma$-hypergroup, when

$$
x \otimes_{\alpha} H=H \otimes_{\alpha} x=H,
$$

for every $\alpha$ of $\Gamma$ and $x \in H$

If for every $\alpha \in \Gamma$, there exists $e_{\alpha} \in H$, such that $x \otimes_{\alpha} e_{\alpha}=e_{\alpha} \otimes_{\alpha} x=x$, for every $x \in H$, then $H$ is called $\Gamma$-semihypergroup with unit.

Example 1. Let $(G, \circ)$ be a group and $\Gamma=\{\alpha, \beta\}$. Then, we define $x \otimes_{\alpha} y=$ $G$ and $x \otimes_{\beta} y=x \circ y$. Thus, $G$ is a $\Gamma$-semihypergroup.

ExAmple 2. Let $A=\left\{A_{g}\right\}_{g \in G}$ be a collection of disjoint and non-empty sets such that $(G, \circ)$ is a semigroup and $\Gamma$ be a non-empty subset of $G$. Then, $T=\bigcup_{g \in G} A_{g}$ is a $\Gamma$-semihypergroup by following hyperoperation:

$$
x_{1} \otimes_{\alpha} x_{2}=A_{g}
$$

where $x_{1} \in A_{g_{1}}, x_{2} \in A_{g_{2}}, g=g_{1} \alpha g_{2}, \alpha \in \Gamma$ and $x_{1}, x_{2} \in G$. We denoted this $\Gamma$-semihypergroup by $T_{A G}$.

Example 3. Let $G=\{a, b\}$ and $\Gamma=\{\alpha, \beta\}$. Then, $G$ is a $\Gamma$-semihypergroup such that hyperoperations are defined as follows:

\begin{tabular}{|c|c|c|}
\hline$\alpha$ & $\mathrm{a}$ & $\mathrm{b}$ \\
\hline $\mathrm{a}$ & $\mathrm{a}$ & $\mathrm{b}$ \\
$\mathrm{b}$ & $\mathrm{b}$ & $\mathrm{a}$ \\
\hline
\end{tabular}$\quad$\begin{tabular}{|c|c|c|}
\hline$\beta$ & $\mathrm{a}$ & $\mathrm{b}$ \\
\hline $\mathrm{a}$ & $\mathrm{b}$ & $\mathrm{a}$ \\
$\mathrm{b}$ & $\mathrm{a}$ & $\mathrm{b}$ \\
\hline
\end{tabular}

Also, $G$ is a $\Gamma$-semihypergroup with unit and $e_{\alpha}=a$ and $e_{\beta}=b$, because for every $z \in G$, we have $a \otimes_{\alpha} z=z \otimes_{\alpha} a=z$ and $b \otimes_{\beta} z=z \otimes_{\beta} b=z$. 
Let $G$ be a $\Gamma$-semihypergroup and the relation $\rho$ defined on

$$
G \times \Gamma=\{(x, \alpha): x \in H, \alpha \in \Gamma\},
$$

as follows:

$$
(x, \alpha) \rho(y, \beta) \Longleftrightarrow \forall z \in G, x \otimes_{\alpha} z=y \otimes_{\beta} z .
$$

This relation is an equivalence. Then, the set $\widehat{G}=\left\{[(x, \alpha)]_{\rho}: x \in H, \alpha \in \Gamma\right\}$ is a semihypergroup by following hyperoperation:

$$
[(x, \alpha)]_{\rho} \circ[(y, \beta)]_{\rho}=\left\{[(z, \beta)]_{\rho}: z \in x \otimes_{\alpha} y\right\} .
$$

Proposition 2.2. Let $\left(G_{1}, \oplus_{\alpha}\right)$ and $\left(G_{2}, \otimes_{\alpha}\right)$ be $\Gamma$-semihypergroups such that $G_{1} \cap G_{2}=\emptyset$. Then, we define a hyperoperation on a new system $G_{1}\left[G_{2}\right]=G_{1} \cup G_{2}$ as follows:

$$
x \odot_{\alpha} y= \begin{cases}x \oplus_{\alpha} y & x, y \in G_{1}, \\ x & x \in G_{2}, y \in G_{1}, \\ y & x \in G_{1}, y \in G_{2} \\ x \otimes_{\alpha} y & x, y \in G_{2}\end{cases}
$$

for all $x, y \in G_{1}\left[G_{2}\right]$ and $\alpha \in \Gamma$.

Proof. The proof is straightforward.

Corollary 2.3. We define the relation $\rho$ on $G_{1}\left[G_{2}\right] \times \Gamma$ as follows:

$$
(x, \alpha) \rho(y, \beta) \Longleftrightarrow \forall z \in G_{1}\left[G_{2}\right], x \odot_{\alpha} z=y \underset{\beta}{\odot} z .
$$

This relation is an equivalence.

Let $G_{1}$ and $G_{2}$ be $\Gamma$-semihypergroups with unit, hence

$$
\left.\widehat{G_{1}\left[G_{2}\right.}\right]=\left\{[(x, \alpha)]_{\rho}: x \in G_{1}\left[G_{2}\right], \alpha \in \Gamma\right\}=\widehat{G_{1}} \cup \widehat{G_{2}}
$$

is a semihypergroup by the following hyperoperation and is called an extension of semihypergroup by semihypergroup.

$$
\left[\left(x_{1}, \alpha_{1}\right)\right]_{\rho} \odot\left[\left(x_{2}, \alpha_{2}\right)\right]_{\rho}= \begin{cases}{\left[\left(x_{1}, \alpha_{1}\right)\right]_{\rho} \circ\left[\left(x_{2}, \alpha_{2}\right)\right]_{\rho}} & {\left[\left(x_{1}, \alpha_{1}\right)\right]_{\rho},\left[\left(x_{2}, \alpha_{2}\right)\right]_{\rho} \in \widehat{G_{1}},} \\ {\left[\left(x_{2}, \alpha_{2}\right)\right]_{\rho}} & {\left[\left(x_{2}, \alpha_{2}\right)\right]_{\rho} \in \widehat{G_{2}},\left[\left(x_{1}, \alpha_{1}\right)\right]_{\rho} \in \widehat{G_{1}},} \\ {\left[\left(x_{1}, \alpha_{1}\right)\right]_{\rho}} & {\left[\left(x_{1}, \alpha_{1}\right)\right]_{\rho} \in \widehat{G_{2}},\left[\left(x_{2}, \alpha_{2}\right)\right]_{\rho} \in \widehat{G_{1}},} \\ {\left[\left(x_{1}, \alpha_{1}\right)\right]_{\rho} \circ\left[\left(x_{2}, \alpha_{2}\right)\right]_{\rho}} & {\left[\left(x_{1}, \alpha_{1}\right)\right]_{\rho},\left[\left(x_{2}, \alpha_{2}\right)\right]_{\rho} \in \widehat{G_{2}} .}\end{cases}
$$


ExAmPLe 4 . Let $G_{1}=\{a, b\}$ be a $\Gamma$-semihypergroup with unit such that $\Gamma=\{\alpha, \beta\}, e_{\alpha}=a, e_{\beta}=b$ and

\begin{tabular}{|c|c|c|}
\hline$\oplus_{\alpha}$ & $\mathrm{a}$ & $\mathrm{b}$ \\
\hline $\mathrm{a}$ & $\mathrm{a}$ & $\mathrm{b}$ \\
$\mathrm{b}$ & $\mathrm{b}$ & $\mathrm{a}$ \\
\hline
\end{tabular}

\begin{tabular}{|c|c|c|}
\hline$\oplus_{\beta}$ & $\mathrm{a}$ & $\mathrm{b}$ \\
\hline $\mathrm{a}$ & $\mathrm{b}$ & $\mathrm{a}$ \\
$\mathrm{b}$ & $\mathrm{a}$ & $\mathrm{b}$ \\
\hline
\end{tabular}

and $G_{2}=\{c, d, e\}$ is a $\Gamma$-semihypergroup with unit, where

\begin{tabular}{|c|c|c|c|}
\hline$\otimes_{\alpha}$ & $\mathrm{c}$ & $\mathrm{d}$ & $\mathrm{e}$ \\
\hline $\mathrm{c}$ & $\mathrm{c}$ & $\{\mathrm{d}, \mathrm{e}\}$ & $\{\mathrm{d}, \mathrm{e}\}$ \\
$\mathrm{d}$ & $\{\mathrm{d}, \mathrm{e}\}$ & $\mathrm{c}$ & $\mathrm{c}$ \\
$\mathrm{e}$ & $\{\mathrm{d}, \mathrm{e}\}$ & $\mathrm{c}$ & $\mathrm{c}$ \\
\hline
\end{tabular}

\begin{tabular}{|c|c|c|c|}
\hline$\otimes_{\beta}$ & $\mathrm{c}$ & $\mathrm{d}$ & $\mathrm{e}$ \\
\hline $\mathrm{c}$ & $\{\mathrm{d}, \mathrm{e}\}$ & $\mathrm{c}$ & $\mathrm{c}$ \\
$\mathrm{d}$ & $\mathrm{c}$ & $\{\mathrm{d}, \mathrm{e}\}$ & $\{\mathrm{d}, \mathrm{e}\}$ \\
$\mathrm{e}$ & $\mathrm{c}$ & $\{\mathrm{d}, \mathrm{e}\}$ & $\{\mathrm{d}, \mathrm{e}\}$ \\
\hline
\end{tabular}

and $e_{\alpha}=c, e_{\beta}=d$. Therefore, the hyperoperations $\odot_{\alpha}$ and $\odot_{\beta}$ definded on $G_{1}\left[G_{2}\right]$ as follows:

\begin{tabular}{|c|c|c|c|c|c|}
\hline$\odot_{\alpha}$ & $\mathrm{a}$ & $\mathrm{b}$ & $\mathrm{c}$ & $\mathrm{d}$ & $\mathrm{e}$ \\
\hline $\mathrm{a}$ & $\mathrm{a}$ & $\mathrm{b}$ & $\mathrm{c}$ & $\mathrm{d}$ & $\mathrm{e}$ \\
$\mathrm{b}$ & $\mathrm{b}$ & $\mathrm{a}$ & $\mathrm{c}$ & $\mathrm{d}$ & $\mathrm{e}$ \\
$\mathrm{c}$ & $\mathrm{c}$ & $\mathrm{c}$ & $\mathrm{c}$ & $\{\mathrm{d}, \mathrm{e}\}$ & $\{\mathrm{d}, \mathrm{e}\}$ \\
$\mathrm{d}$ & $\mathrm{d}$ & $\mathrm{d}$ & $\{\mathrm{d}, \mathrm{e}\}$ & $\mathrm{c}$ & $\mathrm{c}$ \\
$\mathrm{e}$ & $\mathrm{e}$ & $\{\mathrm{d}, \mathrm{e}\}$ & $\mathrm{c}$ & $\mathrm{c}$ & $\mathrm{c}$ \\
\hline
\end{tabular}

\begin{tabular}{|c|c|c|c|c|c|}
\hline$\odot_{\beta}$ & $\mathrm{a}$ & $\mathrm{b}$ & $\mathrm{c}$ & $\mathrm{d}$ & $\mathrm{e}$ \\
\hline $\mathrm{a}$ & $\mathrm{b}$ & $\mathrm{a}$ & $\mathrm{c}$ & $\mathrm{d}$ & $\mathrm{e}$ \\
$\mathrm{b}$ & $\mathrm{a}$ & $\mathrm{b}$ & $\mathrm{c}$ & $\mathrm{d}$ & $\mathrm{e}$ \\
$\mathrm{c}$ & $\mathrm{c}$ & $\mathrm{c}$ & $\{\mathrm{d}, \mathrm{e}\}$ & $\mathrm{c}$ & $\mathrm{c}$ \\
$\mathrm{d}$ & $\mathrm{d}$ & $\mathrm{d}$ & $\mathrm{c}$ & $\{\mathrm{d}, \mathrm{e}\}$ & $\{\mathrm{d}, \mathrm{e}\}$ \\
$\mathrm{e}$ & $\mathrm{e}$ & $\mathrm{e}$ & $\mathrm{c}$ & $\{\mathrm{d}, \mathrm{e}\}$ & $\{\mathrm{d}, \mathrm{e}\}$ \\
\hline
\end{tabular}

But $G_{1}\left[G_{2}\right]$ is not $\Gamma$-semihypergroup, since

$$
\begin{gathered}
\left(c \odot_{\alpha} b\right) \bigodot_{\beta} e=c \bigodot_{\beta} e=c, c \odot_{\alpha}\left(b \odot_{\beta} e\right)=c \odot_{\alpha} e=\{d, e\}, \\
\left(c \odot_{\alpha} b\right) \odot_{\beta} e \neq c \odot_{\alpha}\left(b \odot_{\beta} e\right) .
\end{gathered}
$$

$\widehat{G_{1}}$ and $\widehat{G_{2}}$ are semihypergroups, Also, $\left(\widehat{G_{1}}\left[\widehat{G_{2}}\right], \odot\right)$ is a semihypergroup.

ExAmPle 5. Let $G_{1}=\{a, b, c\}$ and $G_{2}=\{d, e, f\}$ be $\Gamma$-semihypergroups with unit, where $\Gamma=\{\alpha, \beta\}$ and

\begin{tabular}{|c|c|c|c|}
\hline$\oplus_{\alpha}$ & $\mathrm{a}$ & $\mathrm{b}$ & $\mathrm{c}$ \\
\hline $\mathrm{a}$ & $\mathrm{a}$ & $\{\mathrm{b}, \mathrm{c}\}$ & $\{\mathrm{b}, \mathrm{c}\}$ \\
$\mathrm{b}$ & $\{\mathrm{b}, \mathrm{c}\}$ & $\mathrm{a}$ & $\mathrm{a}$ \\
$\mathrm{c}$ & $\{\mathrm{b}, \mathrm{c}\}$ & $\mathrm{a}$ & $\mathrm{a}$ \\
\hline
\end{tabular}

\begin{tabular}{|c|c|c|c|}
\hline$\oplus_{\beta}$ & $\mathrm{a}$ & $\mathrm{b}$ & $\mathrm{c}$ \\
\hline $\mathrm{a}$ & $\{\mathrm{b}, \mathrm{c}\}$ & $\mathrm{a}$ & $\mathrm{a}$ \\
$\mathrm{b}$ & $\mathrm{a}$ & $\{\mathrm{b}, \mathrm{c}\}$ & $\{\mathrm{b}, \mathrm{c}\}$ \\
$\mathrm{c}$ & $\mathrm{a}$ & $\{\mathrm{b}, \mathrm{c}\}$ & $\{\mathrm{b}, \mathrm{c}\}$ \\
\hline
\end{tabular}

\begin{tabular}{|c|c|c|c|}
\hline$\otimes_{\alpha}$ & $\mathrm{d}$ & $\mathrm{e}$ & $\mathrm{f}$ \\
\hline $\mathrm{d}$ & $\mathrm{d}$ & $\{\mathrm{e}, \mathrm{f}\}$ & $\{\mathrm{e}, \mathrm{f}\}$ \\
$\mathrm{e}$ & $\{\mathrm{e}, \mathrm{f}\}$ & $\mathrm{d}$ & $\mathrm{d}$ \\
$\mathrm{f}$ & $\{\mathrm{e}, \mathrm{f}\}$ & $\mathrm{d}$ & $\mathrm{d}$ \\
\hline
\end{tabular}

\begin{tabular}{|c|c|c|c|}
\hline$\otimes_{\beta}$ & $\mathrm{d}$ & $\mathrm{e}$ & $\mathrm{f}$ \\
\hline $\mathrm{d}$ & $\{\mathrm{e}, \mathrm{f}\}$ & $\mathrm{d}$ & $\mathrm{d}$ \\
$\mathrm{e}$ & $\mathrm{d}$ & $\{\mathrm{e}, \mathrm{f}\}$ & $\{\mathrm{e}, \mathrm{f}\}$ \\
$\mathrm{f}$ & $\mathrm{d}$ & $\{\mathrm{e}, \mathrm{f}\}$ & $\{\mathrm{e}, \mathrm{f}\}$ \\
\hline
\end{tabular}

Then, by Proposition 2.2, $\widehat{G}_{1}\left[\widehat{G}_{2}\right]$ is a semihypergroup. 
Example 6. Let $A=\left\{A_{g}\right\}_{g \in G}$ and $B=\left\{B_{k}\right\}_{k \in K}$ be collections of disjoint and non-empty sets such that $(G, \circ)$ and $(K, \circ)$ are semigroups, $G \cap K=\emptyset$ and $\Gamma, \Gamma^{\prime}$ be non-empty subsets of $G$ and $K$, respectively. Then, $H_{1}=\bigcup_{g \in G} A_{g}$ and $H_{2}=\bigcup_{k \in K} B_{k}$ are $\Gamma$-semihypergroup and $\Gamma^{\prime}$-semihypergroup, respectively, by the following hyperoperations:

$$
\begin{gathered}
x_{1}, x_{2} \in H_{1}, \alpha \in \Gamma, x_{1} \otimes_{\alpha} x_{2}=A_{g}: x_{1} \in A_{g_{1}}, x_{2} \in A_{g_{2}}, g=g_{1} \circ \alpha \circ g_{2} . \\
x_{1}, x_{2} \in H_{2}, \alpha^{\prime} \in \Gamma^{\prime}, x_{1} \underset{\alpha^{\prime}}{\otimes_{2}} x_{2}=B_{k}: x_{1} \in B_{k_{1}}, x_{2} \in B_{k_{2}}, k=k_{1} \circ \alpha^{\prime} \circ k_{2} .
\end{gathered}
$$

Note that $H_{1}$ and $H_{2}$ don't have unit. From $G \cap K=\emptyset$, we have $\Gamma \cap \Gamma^{\prime}=\emptyset$, therefore $\Gamma\left[\Gamma^{\prime}\right]=\Gamma \cup \Gamma^{\prime}$. Also, we conclude that $A_{g} \cap B_{k}=\emptyset$. This implies that $H_{1} \cap H_{2}=\emptyset$. So, $\widehat{H_{1}} \cap \widehat{H_{2}}=\emptyset$. Then, $\widehat{H_{1}\left[H_{2}\right]}$ is a semihypergroup but $H_{1}\left[H_{2}\right]$ is not $\Gamma\left[\Gamma^{\prime}\right]$-semihypergroup.

Proposition 2.4. Let $G_{1}$ and $G_{2}$ be $\Gamma$-semihypergroups with unit such that $G_{1} \cap G_{2}=\emptyset$. Then, $\widehat{G_{1}\left[G_{2}\right]}=\widehat{G_{1}}\left[\widehat{G_{2}}\right]$.

Proof. Suppose that $[(x, \alpha)]_{\rho} \in \widehat{G_{1}\left[G_{2}\right]}$. Hence, there exist $y \in G_{1}\left[G_{2}\right]$ and $\beta \in \Gamma$ such that $[(x, \alpha)]_{\rho}=[(y, \beta)]_{\rho}$. This implies that $y \in G_{1}$ or $y \in G_{2}$. Then, $[(y, \beta)]_{\rho} \in \widehat{G_{1}}$ or $[(y, \beta)]_{\rho} \in \widehat{G_{2}}$. So, $[(x, \alpha)]_{\rho}=[(y, \beta)]_{\rho} \in \widehat{G_{1}} \cup \widehat{G_{2}}$ and $\widehat{G_{1}} \cup \widehat{G_{2}}=\widehat{G_{1}}\left[\widehat{G_{2}}\right]$, Also, $\widehat{G_{1}} \cap \widehat{G_{2}}=\emptyset$. Since, $G_{1} \cap G_{2}=\emptyset$. We obtain $\left.\widehat{G_{1}\left[G_{2}\right.}\right] \subseteq \widehat{G_{1}}\left[\widehat{G_{2}}\right]$.

Now, suppose that $[(x, \alpha)]_{\rho} \in \widehat{G_{1}}\left[\widehat{G_{2}}\right]$. Then, $[(x, \alpha)]_{\rho} \in \widehat{G_{1}}$ or $[(x, \alpha)]_{\rho} \in \widehat{G_{2}}$. So, there exist $t_{1} \in G_{1}, \beta_{1} \in \Gamma$ or $t_{2} \in G_{2}, \beta_{2} \in \Gamma$ such that $[(x, \alpha)]_{\rho}=$ $\left[\left(t_{1}, \beta_{1}\right)\right]_{\rho}$ or $[(x, \alpha)]_{\rho}=\left[\left(t_{2}, \beta_{2}\right)\right]_{\rho}$. Hence, for every $z \in G_{1}, x \otimes_{\alpha} z_{1}=t_{1} \otimes_{\beta_{1}} z_{1}$ and for every $z_{2} \in G_{2}, x \otimes_{\alpha} z_{2}=t_{2} \otimes z_{2}$. We set $z_{1}=e_{\alpha}, z_{2}=e_{\alpha}$. Then, $x \in t_{1} \underset{\beta_{1}}{\otimes} e_{\alpha} \subseteq G_{1}$ and $x \in t_{2} \underset{\beta_{2}}{\otimes} e_{\alpha} \subseteq G_{2}$. So, $x \in G_{1}$ or $x \in G_{2}$. We obtain $x \in G_{1}\left[G_{2}\right]$ and $\alpha \in \Gamma$. This means that $\left.[(x, \alpha)]_{\rho} \in \widehat{G_{1}\left[G_{2}\right.}\right]$. Therefore, $\left.\widehat{G_{1}}\left[\widehat{G_{2}}\right] \subseteq \widehat{G_{1}\left[G_{2}\right.}\right]$.

Definition 2.5. Let $G_{1}$ and $G_{2}$ be $\Gamma$-semihypergroups with unit such that $G_{1} \cap G_{2}=\emptyset$ and $A \subseteq G_{1}\left[G_{2}\right], B \subseteq \widehat{G}_{1}\left[\widehat{G}_{2}\right]$. We define

$$
\begin{gathered}
\widehat{A}=\left\{[(x, \alpha)]_{\rho}: x \in A, \alpha \in \Gamma\right\}, \\
B^{\prime}=\left\{x \in G_{1}\left[G_{2}\right]: \exists \alpha \in \Gamma:[(x, \alpha)]_{\rho} \in B\right\} .
\end{gathered}
$$


Proposition 2.6. Let $G_{1}$ and $G_{2}$ be $\Gamma$-semihypergroups with unit such that $G_{1} \cap G_{2}=\emptyset, A, B \in \mathcal{P}^{*}\left(G_{1}\left[G_{2}\right]\right)$, and $C \in \mathcal{P}^{*}\left(\widehat{G}_{1}\left[\widehat{G}_{2}\right]\right)$. Then, the following statements hold:

1) $C \subseteq \widehat{\left(C^{\prime}\right)}$.

And if $A$ and $B$ are $\Gamma$-hyperideals of $G_{1}\left[G_{2}\right]$, then

2) $\widehat{A \cap B}=\widehat{A} \cap \widehat{B}$

3) $\widehat{A \cup B}=\widehat{A} \cup \widehat{B}$

4) $(\widehat{A})^{\prime}=A$,

5) $\widehat{A \otimes B}=\widehat{A} \circ \widehat{B}$.

Proof. The proof is straightforward.

In the Proposition 2.6,[1], in general $C$ is not equal to $\widehat{\left(C^{\prime}\right)}$.

Example 7. In Example 4, let $C=\left\{[(a, \alpha)]_{\rho},[(c, \beta)]_{\rho}\right\} \subseteq \widehat{G_{1}}\left[\widehat{G_{2}}\right]$. Then, $C^{\prime}=\{a, c\}$. This implies that

$$
\widehat{\left(C^{\prime}\right)}=\left\{[(a, \alpha)]_{\rho},[(c, \alpha)]_{\rho},[(a, \beta)]_{\rho},[(c, \beta)]_{\rho}\right\} .
$$

We obtain $C \subseteq \widehat{\left(C^{\prime}\right)}$ but $C \neq \widehat{\left(C^{\prime}\right)}$, because $\widehat{\left(C^{\prime}\right)} \nsubseteq C$.

Proposition 2.7. Let $G_{1}$ and $G_{2}$ be $\Gamma$-semihypergroups with unit such that $G_{1} \cap G_{2}=\emptyset$ and $A, B \in \mathcal{P}^{*}\left(\widehat{G}_{1}\left[\widehat{G}_{2}\right]\right)$ such that $A \cap B \neq \emptyset$, and $C \in$ $\mathcal{P}^{*}\left(G_{1}\left[G_{2}\right]\right)$ be a $\Gamma$-hyperideal of $G_{1}\left[G_{2}\right]$. Then, the following statements hold:

1) $(\widehat{C})^{\prime}=C$;

2) $(A \cap B)^{\prime} \subseteq A^{\prime} \cap B^{\prime}$

3) $(A \cup B)^{\prime}=A^{\prime} \cup B^{\prime}$;

4) $A \subseteq \widehat{\left(A^{\prime}\right)}$.

Proof. The proof is straightforward.

Example 8. In Example 4, we have

$$
\begin{aligned}
\widehat{G_{1}}\left[\widehat{G_{2}}\right]= & \widehat{G_{1}} \cup \widehat{G_{2}} \\
= & \left\{[(a, \alpha)]_{\rho},[(a, \beta)]_{\rho},[(b, \alpha)]_{\rho},[(b, \beta)]_{\rho},\right. \\
& \left.\quad[(c, \alpha)]_{\rho},[(c, \beta)]_{\rho},[(d, \alpha)]_{\rho},[(d, \beta)]_{\rho},[(e, \alpha)]_{\rho},[(e, \beta)]_{\rho}\right\} .
\end{aligned}
$$


Let $A=\left\{[(a, \alpha)]_{\rho},[(b, \beta)]_{\rho}\right\}$ and $B=\left\{[(a, \alpha)]_{\rho},[(b, \alpha)]_{\rho}\right\}$. Then, $A^{\prime}=\{a, b\}$ and $B^{\prime}=\{a, b\}$. So, $A^{\prime} \cap B^{\prime}=\{a, b\}$. Moreover, $A \cap B=\left\{[(a, \alpha)]_{\rho}\right\}$. We obtain $(A \cap B)^{\prime}=\{a\}$. We conclude that $A^{\prime} \cap B^{\prime} \nsubseteq(A \cap B)^{\prime}$.

Also, we have $A^{\prime}=\{a, b\}$. This implies that:

$$
\widehat{\left(A^{\prime}\right)}=\left\{[(a, \alpha)]_{\rho},[(a, \beta)]_{\rho},[(b, \alpha)]_{\rho},[(b, \beta)]_{\rho}\right\} .
$$

So, $A \subseteq \widehat{\left(A^{\prime}\right)}$ but $\widehat{\left(A^{\prime}\right)} \nsubseteq A$.

Proposition 2.8. Let $G_{1}$ and $G_{2}$ be $\Gamma$-semihypergroups with unit such that $G_{1} \cap G_{2}=\emptyset A, B$ are nonempty subsets of $G_{1}\left[G_{2}\right]$ such that $B$ is a right $\Gamma$-hyperideal of $G_{1}\left[G_{2}\right]$. Then,

$$
A \subseteq B \Longleftrightarrow \widehat{A} \subseteq \widehat{B} .
$$

Proof. Assume that $[(x, \alpha)]_{\rho} \in \widehat{A}$, then there exist $y \in A$ and $\beta \in \Gamma$ such that $[(x, \alpha)]_{\rho}=[(y, \beta)]_{\rho}$. Also, we have $y \in A \subseteq B$. So, $y \in B$ and $[(y, \beta)]_{\rho} \in \widehat{B}$. We conclude that $[(x, \alpha)]_{\rho} \in \widehat{B}$ and this means that $\widehat{A} \subseteq \widehat{B}$.

Conversely, let $x \in A$. Then, for every $\alpha \in \Gamma,[(x, \alpha)]_{\rho} \in \widehat{A} \subseteq \widehat{B}$. This implies that $[(x, \alpha)]_{\rho} \in \widehat{B}$. So, there exist $y \in B$ and $\beta \in \Gamma$ such that $[(x, \alpha)]_{\rho}=[(y, \beta)]_{\rho}$. Hence, for every $z$ of $G_{1}\left[G_{2}\right], x \otimes_{\alpha} z=y \underset{\beta}{\otimes_{\beta}} z$ and for every $z \in G_{1}$ or $z \in G_{2}, x \otimes_{\alpha} z=y \otimes_{\beta} z$. If $z \in G_{1}$, we set $z=e_{\alpha}$ (the unit element corresponding $\alpha$ of $G_{1}$ ), then

$$
x=x \otimes_{\alpha} e_{\alpha} \in y \underset{\beta}{\otimes} e_{\alpha} \subseteq B \underset{\Gamma}{\otimes} G_{1} \subseteq B \underset{\Gamma}{\otimes} G_{1}\left[G_{2}\right] \subseteq B .
$$

If $z \in G_{2}$, in the same way, we obtain $x \in B$. We conclude that $A \subseteq B$.

Proposition 2.9. Let $G_{1}$ and $G_{2}$ are $\Gamma$-semihypergroups with unit such that $G_{1} \cap G_{2}=\emptyset$ and $A, B$ be nonempty subsets of $\widehat{G_{1}}\left[\widehat{G_{2}}\right]$. Then,

$$
A \subseteq B \Longrightarrow A^{\prime} \subseteq B^{\prime}
$$

Proof. The proof is straightforward.

Definition 2.10. Let $G_{1}, G_{2}, H_{1}$, and $H_{2}$ be $\Gamma$-semihypergroups such that $G_{1} \cap G_{2}=\emptyset$ and $H_{1} \cap H_{2}=\emptyset$. Then, a map $\Phi: \widehat{G_{1}}\left[\widehat{G_{2}}\right] \longrightarrow \widehat{H_{1}}\left[\widehat{H_{2}}\right]$ is called a homomorphism between semihypergroups, if for all $\left[\left(x_{1}, \alpha\right)\right]_{\rho},\left[\left(x_{2}, \beta\right)\right]_{\rho} \in$ $\widehat{G_{1}}\left[\widehat{G_{2}}\right]$,

$$
\Phi\left(\left[\left(x_{1}, \alpha\right)\right]_{\rho} \circ\left[\left(x_{2}, \beta\right)\right]_{\rho}\right)=\Phi\left(\left[\left(x_{1}, \alpha\right)\right]_{\rho}\right) \circ \Phi\left(\left[\left(x_{2}, \beta\right)\right]_{\rho}\right) .
$$


Definition 2.11. Let $G_{1}, G_{2}, H_{1}$, and $H_{2}$ be $\Gamma$-semihypergroups such that $G_{1} \cap G_{2}=\emptyset$ and $H_{1} \cap H_{2}=\emptyset$. Then, we define a hyperoperation $\underset{\left(\alpha_{1}, \alpha_{2}\right)}{\otimes}$ on $G_{1}\left[G_{2}\right] \times H_{1}\left[H_{2}\right]$ as follows:

$$
\begin{gathered}
\underset{\left(\alpha_{1}, \alpha_{2}\right)}{\otimes}:\left(G_{1}\left[G_{2}\right] \times H_{1}\left[H_{2}\right]\right) \times\left(G_{1}\left[G_{2}\right] \times H_{1}\left[H_{2}\right]\right) \longrightarrow \mathcal{P}^{*}\left(G_{1}\left[G_{2}\right] \times H_{1}\left[H_{2}\right],\right) \\
\left(x_{1}, y_{1}\right) \underset{\left(\alpha_{1}, \alpha_{2}\right)}{\otimes}\left(x_{2}, y_{2}\right)=\left\{\left(t_{1}, t_{2}\right): t_{1} \in x_{1} \underset{\alpha_{1}}{\otimes} x_{2}, t_{2} \in y_{1} \underset{\alpha_{2}}{\otimes} y_{2}\right\}
\end{gathered}
$$

where $\underset{\alpha_{1}}{\otimes}: G_{1}\left[G_{2}\right] \times G_{1}\left[G_{2}\right] \longrightarrow \mathcal{P}^{*}\left(G_{1}\left[G_{2}\right]\right)$ and $\underset{\alpha_{2}}{\otimes}: H_{1}\left[H_{2}\right] \times H_{1}\left[H_{2}\right] \longrightarrow$ $\mathcal{P}^{*}\left(H_{1}\left[H_{2}\right]\right)$.

Also, we define

$$
\begin{gathered}
G_{1}\left[G_{2} \widehat{{ }^{\prime} H_{1}}\left[H_{2}\right]=\left\{\left[\left(\left(x_{1}, x_{2}\right),\left(\alpha_{1}, \alpha_{2}\right)\right)\right]_{\rho_{G_{1}\left[G_{2}\right] \times H_{1}\left[H_{2}\right]}} .\right.\right. \\
\left.:\left(x_{1}, x_{2}\right) \in G_{1}\left[G_{2}\right] \times H_{1}\left[H_{2}\right], \alpha_{1}, \alpha_{2} \in \Gamma\right\} .
\end{gathered}
$$

Theorem 2.12. Let $G_{1}, G_{2}, H_{1}$, and $H_{2}$ be $\Gamma$-semimihypergroups s with unit such that $G_{1} \cap G_{2}=\emptyset$ and $H_{1} \cap H_{2}=\emptyset$. Then,

$$
\left(G_{1}\left[G_{2}\right] \times H_{1}\left[H_{2}\right]\right) \cong \widehat{G_{1}}\left[\widehat{G_{2}}\right] \times \widehat{H_{1}}\left[\widehat{H_{2}}\right] .
$$

Proof. Suppose that $\rho_{G_{1}\left[G_{2}\right] \times H_{1}\left[H_{2}\right]}, \rho_{G_{1}\left[G_{2}\right]}$, and $\rho_{H_{1}\left[H_{2}\right]}$ are equivalence relations defined on $G_{1}\left[G_{2}\right] \times H_{1}\left[H_{2}\right], G_{1}\left[G_{2}\right]$, and $H_{1}\left[H_{2}\right]$, respectively. Let $\Psi:\left(G_{1}\left[G_{2}\right] \times H_{1}\left[H_{2}\right]\right) \longrightarrow \widehat{G_{1}}\left[\widehat{G_{2}}\right] \times \widehat{H_{1}}\left[\widehat{H_{2}}\right]$ be defined as follows:

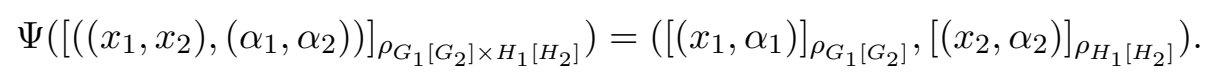

Hence,

$$
\left[\left(\left(x_{1}, x_{2}\right),\left(\alpha_{1}, \alpha_{2}\right)\right)\right]_{\rho_{G_{1}\left[G_{2}\right] \times H_{1}\left[H_{2}\right]}}=\left[\left(\left(y_{1}, y_{2}\right),\left(\beta_{1}, \beta_{2}\right)\right)\right]_{\left.\rho_{G_{1}\left[G_{2}\right] \times H_{1}\left[H_{2}\right]}\right]},
$$

if and only if for every $\left(z_{1}, z_{2}\right) \in G_{1}\left[G_{2}\right] \times H_{1}\left[H_{2}\right]$,

$$
\left(x_{1}, x_{2}\right) \underset{\left(\alpha_{1}, \alpha_{2}\right)}{\otimes}\left(z_{1}, z_{2}\right)=\left(y_{1}, y_{2}\right) \underset{\left(\beta_{1}, \beta_{2}\right)}{\otimes}\left(z_{1}, z_{2}\right),
$$

if and only if for every $z_{1} \in G_{1}\left[G_{2}\right]$ and $z_{2} \in H_{1}\left[H_{2}\right]$,

$$
x_{1} \underset{\alpha_{1}}{\otimes} z_{1}=y_{1} \underset{\beta_{1}}{\otimes} z_{1}, \quad x_{2} \underset{\alpha_{2}}{\otimes} z_{2}=y_{2} \underset{\beta_{2}}{\otimes} z_{2},
$$

if and only if

$$
\left[\left(x_{1}, \alpha_{1}\right)\right]_{\rho_{G_{1}\left[G_{2}\right]}}=\left[\left(y_{1}, \beta_{1}\right)\right]_{\rho_{G_{1}\left[G_{2}\right]}}, \quad\left[\left(x_{2}, \alpha_{2}\right)\right]_{\rho_{H_{1}\left[H_{2}\right]}}=\left[\left(y_{2}, \beta_{2}\right)\right]_{\rho_{H_{1}\left[H_{2}\right]}} .
$$


Hence, $\Psi$ is well-defined and one to one. If $\left(\left[\left(x_{1}, \alpha_{1}\right)\right]_{\left.\rho_{G_{1}\left[G_{2}\right]}\right]}\left[\left(x_{2}, \alpha_{2}\right)\right]_{\rho_{H_{1}\left[H_{2}\right]}}\right) \in$ $\widehat{G_{1}}\left[\widehat{G_{2}}\right] \times \widehat{H_{1}}\left[\widehat{H_{2}}\right]$, then

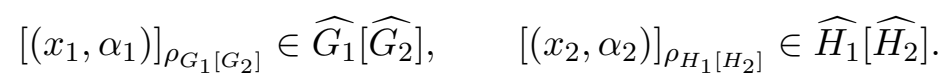

We obtain $\left[\left(x_{1}, \alpha_{1}\right)\right]_{\rho_{G_{1}\left[G_{2}\right]}} \in \widehat{G_{1}}$ or $\left[\left(x_{1}, \alpha_{1}\right)\right]_{\rho_{G_{1}\left[G_{2}\right]}} \in \widehat{G_{2}}$ and $\left[\left(x_{2}, \alpha_{2}\right)\right]_{\rho_{H_{1}\left[H_{2}\right]}} \in$

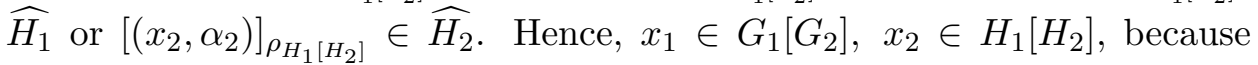
$G_{1}, G_{2}, H_{1}$, and $H_{2}$ are $\Gamma$-semihypergroup with unit. This implies that

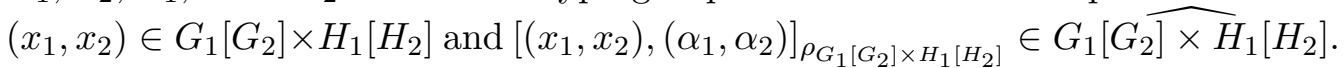
So, $\Psi$ is onto. Now, we show that $\Psi$ is homomorphism of semihypergroups:

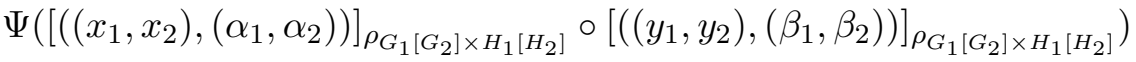

$$
\begin{aligned}
& =\Psi\left(\left[\left(\left(x_{1}, x_{2}\right) \underset{\left(\alpha_{1}, \alpha_{2}\right.}{\otimes}\left(y_{1}, y_{2}\right),\left(\beta_{1}, \beta_{2}\right)\right)\right]_{\left.\rho_{G_{1}\left[G_{2}\right] \times H_{1}\left[H_{2}\right]}\right)}\right. \\
& =\Psi\left(\left[\left(\left(x_{1} \underset{\alpha_{11}}{y}, x_{2} \underset{\alpha_{2}}{\otimes} y_{2}\right),\left(\beta_{1}, \beta_{2}\right)\right)\right]_{\left.\rho_{G_{1}\left[G_{2}\right] \times H_{1}\left[H_{2}\right]}\right)}\right. \\
& =\left(\left[\left(x_{1} \underset{\alpha_{1}}{\otimes} y_{1}, \beta_{1}\right)\right]_{\rho_{G_{1}\left[G_{2}\right]}},\left[\left(x_{2} \underset{\alpha_{2}}{\otimes} y_{2}, \beta_{2}\right)\right]_{\rho_{H_{1}\left[H_{2}\right]}}\right) \\
& =\left(\left[\left(x_{1}, \alpha_{1}\right)\right]_{\rho_{G_{1}\left[G_{2}\right]}} \circ\left[\left(y_{1}, \beta_{1}\right)\right]_{\rho_{G_{1}\left[G_{2}\right]}},\left[\left(x_{2}, \alpha_{2}\right)\right]_{\rho_{H_{1}\left[H_{2}\right]}} \circ\left[\left(y_{2}, \beta_{2}\right)\right]_{\rho_{H_{1}\left[H_{2}\right]}}\right)
\end{aligned}
$$

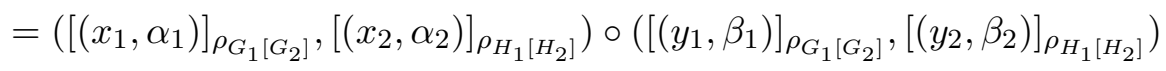

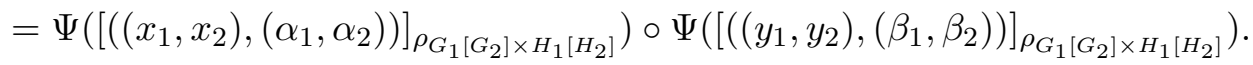

This complete the proof.

\section{Relations between rough soft semihypergroups and their associated rough soft sets}

In this section, first we define rough semihypergroups, soft semihypergroups and prove that there is a relation between them and associated rough and soft sets. After that we define rough soft semihypergroups by defining a regular relation and we show that there are relations between rough soft semihypergroups and their associated rough soft sets.

Definition 3.1. Let $G_{1}$ and $G_{2}$ be $\Gamma$-semihypergroups such that $G_{1} \cap G_{2}=\emptyset$ and $\Theta$ be an equivalence relation on $G_{1}\left[G_{2}\right]$. Then, we define the relation $\widehat{\Theta}$ on $\widehat{G_{1}}\left[\widehat{G_{2}}\right]$ as follows:

$$
[(x, \alpha)]_{\rho} \widehat{\Theta}[(y, \beta)]_{\rho} \Longleftrightarrow\left(x \otimes_{\alpha} z\right) \bar{\Theta}(y \underset{\beta}{\otimes} z) \quad \text { for all } z \in G_{1}\left[G_{2}\right] .
$$


Definition 3.2. Let $G_{1}$ and $G_{2}$ be $\Gamma$-semihypergroups such that $G_{1} \cap G_{2}=\emptyset$ and $\Theta$ be an equivalence relation on $G_{1}\left[G_{2}\right]$. Then, the upper and lower approximations of $A \subseteq G_{1}\left[G_{2}\right]$ defined by

$$
\begin{gathered}
\overline{A p r}_{\Theta}(A)=\left\{x \in G_{1}\left[G_{2}\right]:[x]_{\Theta} \cap A \neq \emptyset\right\}, \\
\operatorname{Apr}_{\Theta}(A)=\left\{x \in G_{1}\left[G_{2}\right]:[x]_{\Theta} \subseteq A\right\} .
\end{gathered}
$$

EXAmPle 9. Let $G_{1}=\{a, b, c\}$ be a $\Gamma$-semihypergroup such that $\Gamma=\{\alpha, \beta\}$ and

\begin{tabular}{|c|c|c|c|}
\hline$\alpha$ & $\mathrm{a}$ & $\mathrm{b}$ & $\mathrm{c}$ \\
\hline $\mathrm{a}$ & $\mathrm{a}$ & $\{\mathrm{b}, \mathrm{c}\}$ & $\{\mathrm{b}, \mathrm{c}\}$ \\
$\mathrm{b}$ & $\{\mathrm{b}, \mathrm{c}\}$ & $\mathrm{a}$ & $\mathrm{a}$ \\
$\mathrm{c}$ & $\{\mathrm{b}, \mathrm{c}\}$ & $\mathrm{a}$ & $\mathrm{a}$ \\
\hline
\end{tabular}

\begin{tabular}{|c|c|c|c|}
\hline$\beta$ & $\mathrm{a}$ & $\mathrm{b}$ & $\mathrm{c}$ \\
\hline $\mathrm{a}$ & $\{\mathrm{b}, \mathrm{c}\}$ & $\mathrm{a}$ & $\mathrm{a}$ \\
$\mathrm{b}$ & $\mathrm{a}$ & $\{\mathrm{b}, \mathrm{c}\}$ & $\{\mathrm{b}, \mathrm{c}\}$ \\
$\mathrm{c}$ & $\mathrm{a}$ & $\{\mathrm{b}, \mathrm{c}\}$ & $\{\mathrm{b}, \mathrm{c}\}$ \\
\hline
\end{tabular}

and $G_{2}=\left\{\left(\begin{array}{lll}1 & 2 & 3\end{array}\right),\left(\begin{array}{lll}1 & 3 & 2\end{array}\right),(1)\right\}$ (as a subset of the symmetric group of degree 3 ) be a $\Gamma$-semihypergroup such that $\Gamma=\{\cdot\}$, where ". " is the multiplication of $S_{3}$. Then, we define an equivalence relation $\Theta$ on $G_{1}\left[G_{2}\right]=$ $\left\{a, b, c,\left(\begin{array}{lll}1 & 2 & 3\end{array}\right),\left(\begin{array}{ll}1 & 3\end{array}\right),(1)\right\}$ as follows:

$$
\begin{aligned}
x \Theta y \Longleftrightarrow & \text { if } x, y \in G_{1}, \exists \gamma \in \Gamma: x \in \underset{\gamma}{\otimes} y, \\
& \text { if } x, y \in G_{2}, x=y .
\end{aligned}
$$

Thus, we obtain the equivalence classes of $G_{1}\left[G_{2}\right]$ w.r.t $\Theta$ as follows:

$$
\begin{gathered}
{[a]_{\Theta}=[b]_{\Theta}=[c]_{\Theta}=G_{1},} \\
{\left[\left(\begin{array}{lll}
1 & 2 & 3
\end{array}\right)\right]_{\Theta}=\left\{\left(\begin{array}{lll}
1 & 2 & 3
\end{array}\right)\right\},\left[\left(\begin{array}{lll}
1 & 3 & 2
\end{array}\right)\right]_{\Theta}=\left\{\left(\begin{array}{lll}
1 & 3 & 2
\end{array}\right)\right\},[(1)]_{\Theta}=\{(1)\} .}
\end{gathered}
$$

Moreover,

$$
\begin{gathered}
\widehat{G_{1}}\left[\widehat{G_{2}}\right]=\left\{[(a, \alpha)]_{\rho},[(a, \beta)]_{\rho},[(b, \alpha)]_{\rho},[(b, \beta)]_{\rho},[(c, \alpha)]_{\rho},[(c, \beta)]_{\rho},[((123), \cdot)]_{\rho}, .\right. \\
\left.[((132), \cdot)]_{\rho},[((1), \cdot)]_{\rho}\right\}
\end{gathered}
$$

is a semihypergroup. By the Definition 3.1, the equivalence classes of $\widehat{\Theta}$ are as follows:

$$
\begin{gathered}
{\left[\left([(a, \alpha)]_{\rho}\right)\right]_{\widehat{\Theta}}=\left[\left([(b, \alpha)]_{\rho}\right)\right]_{\widehat{\Theta}}=\left[\left([(c, \alpha)]_{\rho}\right)\right]_{\widehat{\Theta}}=\left[\left([(a, \beta)]_{\rho}\right)\right]_{\widehat{\Theta}}} \\
=\left[\left([(b, \beta)]_{\rho}\right)\right]_{\widehat{\Theta}}=\left[\left([(c, \beta)]_{\rho}\right)\right]_{\widehat{\Theta}}=\widehat{G_{1}} . \\
{\left[\left([((123), \cdot)]_{\rho}\right)\right]_{\widehat{\Theta}}=\left\{[((123), \cdot)]_{\rho}\right\},\left[\left([((132), \cdot)]_{\rho}\right)\right]_{\widehat{\Theta}}} \\
=\left\{[((132), \cdot)]_{\rho}\right\},\left[\left([((1), \cdot)]_{\rho}\right)\right]_{\widehat{\Theta}}=\left\{[((1), \cdot)]_{\rho}\right\} .
\end{gathered}
$$


ExAMPLE 10. Let $G_{1}$ and $G_{2}$ be canonical hypergroups such that $G_{1} \cap G_{2}=$ $\emptyset$ and $\left\{X_{g}\right\}_{g \in G_{1}\left[G_{2}\right]}$ be collection of disjoint nonempty sets and $\Gamma_{1} \subseteq G_{1}$, $\Gamma_{2} \subseteq G_{2}, H_{1}=\bigcup_{g \in G_{1}} X_{g}$, and $H_{2}=\bigcup_{g \in G_{2}} X_{g}$. Then, $H_{1}$ and $H_{2}$ are $\Gamma_{1^{-}}$ semihypergroup and $\Gamma_{2}$-semihypergroup, respectively by

$$
x_{1} \odot_{\alpha} x_{2}=X_{g}: x_{1} \in X_{g_{1}}, x_{2} \in X_{g_{2}}, g \in g_{1} \otimes_{\alpha_{1}} g_{2}, \alpha_{1} \in \Gamma_{1},
$$

and

$$
x_{1} \odot_{\alpha_{2}} x_{2}=X_{g}: x_{1} \in X_{g_{1}}, x_{2} \in X_{g_{2}}, g \in g_{1} \otimes_{\alpha_{2}} g_{2}, \alpha_{2} \in \Gamma_{2} .
$$

We define an equivalence relation $\Theta$ on $H_{1}\left[H_{2}\right]$ as follows:

$$
x_{1} \Theta x_{2} \Longleftrightarrow \exists 1 \leq i \leq 2, g_{i} \in G_{i}:\left\{x_{1}, x_{2}\right\} \subseteq X_{g_{i}} .
$$

For every $A \subseteq H_{1}\left[H_{2}\right]$, we have

$$
\begin{aligned}
\overline{\operatorname{Apr}}_{\Theta}(A) & =\left\{x \in H_{1}\left[H_{2}\right]:[x]_{\Theta} \cap A \neq \emptyset\right\} \\
& =\left\{x \in H_{1}\left[H_{2}\right]: \exists t \in[x]_{\Theta} \cap A\right\} \\
& =\left\{x \in H_{1}\left[H_{2}\right]: t \in[x]_{\Theta}, t \in A\right\} \\
& =\left\{x \in H_{1}\left[H_{2}\right]: \exists 1 \leq i \leq 2, g_{i} \in G_{i}:\{t, x\} \subseteq X_{g_{i}}, t \in A\right\} \\
& =\left\{x \in H_{1}\left[H_{2}\right]: \exists 1 \leq i \leq 2, g_{i} \in G_{i}: t \in X_{g_{i}} \cap A\right\} \\
& =\left\{x \in H_{1}\left[H_{2}\right]: \exists 1 \leq i \leq 2, g_{i} \in G_{i}: X_{g_{i}} \cap A \neq \emptyset\right\}, \\
\underline{A p r}_{\Theta}(A) & =\left\{x \in H_{1}\left[H_{2}\right]:[x]_{\Theta} \subseteq A\right\} \\
& =\left\{x \in H_{1}\left[H_{2}\right]: \forall t \in[x]_{\Theta} \Rightarrow t \in A\right\} \\
& =\left\{x \in H_{1}\left[H_{2}\right]: \exists 1 \leq i \leq 2, g_{i} \in G_{i}:\{t, x\} \subseteq X_{g_{i}}, t \in A\right\} \\
& =\left\{x \in H_{1}\left[H_{2}\right]: \exists 1 \leq i \leq 2, g_{i} \in G_{i}: t \in X_{g_{i}} \Rightarrow t \in A\right\} \\
& =\left\{x \in H_{1}\left[H_{2}\right]: \exists 1 \leq i \leq 2, g_{i} \in G_{i}: X_{g_{i}} \subseteq A\right\} .
\end{aligned}
$$

Definition 3.3. Let $\Theta$ be an equivalence relation on semihypergroup $\widehat{G_{1}}\left[\widehat{G_{2}}\right]$. Then, we define the relation $\Theta^{\prime}$ on $G_{1}\left[G_{2}\right]$ as follows:

$$
x \Theta^{\prime} y \Longleftrightarrow[(x, \Gamma)]_{\rho} \bar{\Theta}[(y, \Gamma)]_{\rho},
$$

where $x, y \in G_{1}\left[G_{2}\right]$. 
ExAMPLE 11. Let $H_{1}$ and $H_{2}$ be $\Gamma_{1}$-semihypergroup and $\Gamma_{2}$-semihypergroup, respectively, as defined in Example 10. Suppose that $\left\{X_{g}\right\}_{g \in G_{1}\left[G_{2}\right]}$ is a collection of disjoint hyperideals of $H_{1}\left[H_{2}\right]$. Hence, $\left(\widehat{H_{1}}\left[\widehat{H_{2}}\right]\right.$, ○) is a semihypergroup. We define an equivalence relation $\Theta$ on $\widehat{H_{1}}\left[\widehat{H_{2}}\right]$ as follows:

$$
[(x, \alpha)]_{\rho} \Theta[(y, \beta)]_{\rho} \Longleftrightarrow \exists 1 \leq i \leq 2, g_{i} \in G_{i}:\left\{[(x, \alpha)]_{\rho},[(y, \beta)]_{\rho}\right\} \subseteq \widehat{X_{g_{i}}} .
$$

We obtain

$$
x \Theta^{\prime} y \Longleftrightarrow \exists 1 \leq i \leq 2, g_{i} \in G_{i}:\{x, y\} \subseteq X_{g_{i}} .
$$

Because

$$
\begin{aligned}
x \Theta^{\prime} y & \Leftrightarrow \exists \alpha \in \Gamma:[(x, \alpha)]_{\rho} \Theta[(y, \alpha)]_{\rho} \\
& \Leftrightarrow \exists \alpha \in \Gamma, 1 \leq i \leq 2, g_{i} \in G_{i}:\left\{[(x, \alpha)]_{\rho},[(y, \alpha)]_{\rho}\right\} \subseteq \widehat{X_{g_{i}}} \\
& \Leftrightarrow \exists \alpha, \alpha^{\prime}, \beta^{\prime} \in \Gamma, 1 \leq i \leq 2, g_{i} \in G_{i}, x^{\prime}, y^{\prime} \in X_{g_{i}}: \\
& {[(x, \alpha)]_{\rho}=\left[\left(x^{\prime}, \alpha^{\prime}\right)\right]_{\rho},[(y, \alpha)]_{\rho}=\left[\left(y^{\prime}, \beta^{\prime}\right)\right]_{\rho} } \\
& \Leftrightarrow \exists \alpha, \alpha^{\prime}, \beta^{\prime} \in \Gamma, 1 \leq i \leq 2, g_{i} \in G_{i}, x^{\prime}, y^{\prime} \in X_{g_{i}}, z \in H_{1}\left[H_{2}\right]: \\
& x \otimes_{\alpha} z=x^{\prime} \otimes_{\alpha^{\prime}} z, y \otimes_{\alpha} z=y_{\beta^{\prime}}^{\otimes^{\prime}} z \\
& \Leftrightarrow \exists \alpha, \alpha^{\prime}, \beta^{\prime} \in \Gamma, 1 \leq i \leq 2, g_{i} \in G_{i}, x^{\prime}, y^{\prime} \in X_{g_{i}}: \\
& x \otimes_{\alpha} e_{\alpha}=x^{\prime} \otimes e_{\alpha}^{\prime}, y \otimes_{\alpha} e_{\alpha}=y_{\beta^{\prime}}^{\otimes} e_{\alpha} \\
& \Leftrightarrow \exists 1 \leq i \leq 2, g_{i} \in G_{i}: x \in x^{\prime} \underset{\alpha^{\prime}}{\otimes} e_{\alpha} \subseteq X_{g_{i}} \underset{\Gamma}{\otimes} H_{1}\left[H_{2}\right] \subseteq X_{g_{i}}, \\
& y \in y^{\prime} \otimes_{\beta^{\prime}}^{\otimes} e_{\alpha} \subseteq X_{g_{i}} \underset{\Gamma}{\otimes} H_{1}\left[H_{2}\right] \subseteq X_{g_{i}} \\
& \Leftrightarrow \exists 1 \leq i \leq 2, g_{i} \in G_{i}:\{x, y\} \subseteq X_{g_{i}} .
\end{aligned}
$$

Proposition 3.4. Let $\Theta$ be an regular equivalence relation on $G_{1}\left[G_{2}\right]$. Then, for every $[(y, \beta)]_{\rho} \in \widehat{G}_{1}\left[\widehat{G_{2}}\right]$,

$$
\left[\left([(y, \beta)]_{\rho}\right)\right]_{\widehat{\Theta}} \subseteq \widehat{[y]_{\Theta}}
$$

Proof. The proof is straightforward.

Proposition 3.5. Let $G_{1}$ and $G_{2}$ be $\Gamma$-semihypergroups with unit such that $G_{1} \cap G_{2}=\emptyset$ and $\Theta$ be a regular equivalence relation on $G_{1}\left[G_{2}\right]$, and $A$ be a right $\Gamma$-hyperideal of $G_{1}\left[G_{2}\right]$. Then,

$$
\widehat{A p r_{\Theta}}(A)=\underline{A p r} r_{\widehat{\Theta}}(\widehat{A}) \text {. }
$$


Proof. The proof is straightforward.

Theorem 3.6. Let $G_{1}$ and $G_{2}$ be $\Gamma$-semihypergroups with unit such that $G_{1} \cap G_{2}=\emptyset$ and $\Theta$ be a regular equivalence relation on $\widehat{G_{1}}\left[\widehat{G_{2}}\right]$ and $A$ be a right hyperideal of $\widehat{G_{1}}\left[\widehat{G_{2}}\right]$. Then,

$$
\left(\underline{A p r}_{\Theta}(A)\right)^{\prime} \subseteq \underline{A p r}_{\Theta^{\prime}} A^{\prime} .
$$

Proof. Suppose that $x \in\left(\underline{A p r}_{\Theta} A\right)^{\prime}$. There exists $\alpha \in \Gamma$ such that $[(x, \alpha)]_{\rho} \in$ $\underline{\operatorname{Apr}}_{\Theta} A$. Hence $\left[\left([(x, \alpha)]_{\rho}\right)\right]_{\Theta} \subseteq A$. By Proposition 2.9, we conclude that $\left[\left([(x, \alpha)]_{\rho}\right)\right]_{\Theta}^{\prime} \subseteq A^{\prime}$. Let $y \in[x]_{\Theta^{\prime}}$. Then, $y \Theta^{\prime} x$ and $[(y, \Gamma)]_{\rho} \bar{\Theta}[(x, \Gamma)]_{\rho}$. For $\alpha \in \Gamma$, there exists $\beta \in \Gamma$ such that $[(y, \beta)]_{\rho} \Theta[(x, \alpha)]_{\rho}$ and implies that $[(y, \beta)]_{\rho} \in\left[\left([(x, \alpha)]_{\rho}\right)\right]_{\Theta}$. This implies that $y \in\left[\left([(x, \alpha)]_{\rho}\right)\right]_{\Theta}^{\prime}$. we obtain $[x]_{\Theta^{\prime}} \subseteq\left[\left([(x, \alpha)]_{\rho}\right)\right]_{\Theta}^{\prime}$ and we have $\left[\left([(x, \alpha)]_{\rho}\right)\right]_{\Theta}^{\prime} \subseteq A^{\prime}$. Hence, $[x]_{\Theta^{\prime}} \subseteq A^{\prime}$ and we obtain $x \in \underline{A p r}_{\Theta^{\prime}}\left(A^{\prime}\right)$. Therefore, $\left(\underline{A p r}_{\Theta}(A)\right)^{\prime} \subseteq \underline{A p r} \underline{\Theta}^{\prime} A^{\prime}$.

Proposition 3.7. Let $G_{1}$ and $G_{2}$ be $\Gamma$-semihypergroups with unit such that $G_{1} \cap G_{2}=\emptyset$, and $\Theta$ be a regular equivalence relation on $G_{1}\left[G_{2}\right]$, and $A$ be a $\Gamma$-hyperideal of $G_{1}\left[G_{2}\right]$. Then,

$$
\widehat{\operatorname{Apr}_{\Theta}(A)}=\overline{A p r}_{\widehat{\Theta}}(\widehat{A}) .
$$

Proof. The proof is straightforward.

Theorem 3.8. Let $G_{1}$ and $G_{2}$ be $\Gamma$-semihypergroups with unit such that $G_{1} \cap G_{2}=\emptyset$ and $\Theta$ be a regular equivalence relation on $\widehat{G_{1}}\left[\widehat{G_{2}}\right]$, and $A$ be a $\Gamma$-hyperideal of $\widehat{G_{1}}\left[\widehat{G_{2}}\right]$. Then,

$$
\left(\overline{A p r}_{\Theta} A\right)^{\prime}=\overline{A p r}_{\Theta^{\prime}} A^{\prime} .
$$

Proof. Let $x \in\left(\overline{A p r}_{\Theta} A\right)^{\prime}$. There exists $\alpha \in \Gamma$ such that $[(x, \alpha)]_{\rho} \in \overline{A p r}_{\Theta}(A)$. Hence, $\left[\left([(x, \alpha)]_{\rho}\right)\right]_{\Theta} \cap A \neq \emptyset$ and there exists $[(y, \beta)]_{\rho} \in\left[\left([(x, \alpha)]_{\rho}\right)\right]_{\Theta} \cap A$. So, $[(y, \beta)]_{\rho} \Theta[(x, \alpha)]_{\rho}$ and $y \in A^{\prime}$. This implies that $y \Theta^{\prime} x$. We obtain $y \in[x]_{\Theta^{\prime}} \cap A^{\prime}$. This means that $[x]_{\Theta^{\prime}} \cap A^{\prime} \neq \emptyset$. Thus, $x \in \overline{A p r}_{\Theta^{\prime}} A^{\prime}$. We conclude that

$$
\left(\overline{A p r}_{\Theta} A\right)^{\prime} \subseteq \overline{A p r}_{\Theta^{\prime}} A^{\prime}
$$

Conversely, let $x \in \overline{A p r}_{\Theta^{\prime}} A^{\prime}$. Then, $[x]_{\Theta^{\prime}} \cap A^{\prime} \neq \emptyset$. Hence, there exists $y \in[x]_{\Theta^{\prime}} \cap A^{\prime}$. Thus, $y \in[x]_{\Theta^{\prime}}$ and $y \in A^{\prime}$. There exists $\alpha \in \Gamma$ such that $[(y, \alpha)]_{\rho} \in A$ and we have $[(y, \Gamma)]_{\rho} \bar{\Theta}[(x, \Gamma)]_{\rho}$. For $\alpha \in \Gamma$, there exists $\beta \in \Gamma$ such that $[(y, \alpha)]_{\rho} \in\left[\left([(x, \beta)]_{\rho}\right)\right]_{\Theta}$. We conclude that $\left[\left([(x, \beta)]_{\rho}\right)\right]_{\Theta} \cap A \neq \emptyset$. This means that $[(x, \beta)]_{\rho} \in \overline{A p r}_{\Theta} A$. We obtain $x \in\left(\overline{A p r}_{\Theta} A\right)^{\prime}$ and we have $\overline{A p r}_{\Theta} A^{\prime} \subseteq\left(\overline{A p r}_{\Theta} A\right)^{\prime}$. 
Proposition 3.9. Let $G_{1}$ and $G_{2}$ be $\Gamma$-semihypergroups such that $G_{1} \cap G_{2}=$ $\emptyset$ and $\Theta$ be a regular relation on $G_{1}\left[G_{2}\right]$. Then, $\left[G_{1}\left[G_{2}\right]: \Theta\right]=\left\{[x]_{\Theta}: x \in\right.$ $\left.G_{1}\left[G_{2}\right]\right\}$ is a $\Gamma$-hypergroupoid by the following hyperoperation:

$$
\left[x_{1}\right]_{\Theta} \underset{\alpha}{\oplus}\left[x_{2}\right]_{\Theta}=\left\{[z]_{\Theta}: z \in x_{1} \otimes_{\alpha} x_{2}\right\} .
$$

Proof. The proof is straightforward.

There is a connection between a regular relation $\Theta$ on $\widehat{G_{1}}\left[\widehat{G_{2}}\right]$ and the regular relation $\Theta^{\prime}$ on associated $\Gamma$-hypergroupoid $G_{1}\left[G_{2}\right]$ as follows:

Proposition 3.10. Let $\Theta$ be a regular equivalence relation on $G_{1}\left[G_{2}\right]$. Then $\left[\left(\left[x_{1}\right]_{\Theta}, \alpha\right)\right]_{\rho}=\left[\left(\left[x_{2}\right]_{\Theta}, \beta\right)\right]_{\rho}$ if and only if $\left[\left(x_{1}, \alpha\right)\right]_{\rho} \widehat{\Theta}\left[\left(x_{2}, \beta\right)\right]_{\rho}$.

Proof. The proof is straightforward.

Proposition 3.11. Let $\Theta$ be a regular relation on semihypergroup $\widehat{G_{1}}\left[\widehat{G_{2}}\right]$. Then,

$$
\left[\left([(x, \alpha)]_{\rho}\right)\right]_{\Theta}=\left[\left([(y, \alpha)]_{\rho}\right)\right]_{\Theta} \Longleftrightarrow\left[\left([x]_{\Theta^{\prime}}, \alpha\right)\right]_{\rho}=\left[\left([y]_{\Theta^{\prime}}, \alpha\right)\right]_{\rho} .
$$

Proof. Suppose that $[(x, \alpha)]_{\rho},[(x, \beta)]_{\rho} \in \widehat{G_{1}}\left[\widehat{G_{2}}\right]$. Hence,

$$
\begin{aligned}
{\left[\left([(x, \alpha)]_{\rho}\right)\right]_{\Theta}=\left[\left([(y, \beta)]_{\rho}\right)\right]_{\Theta} } & \Longleftrightarrow[(x, \alpha)]_{\rho} \Theta[(y, \beta)]_{\rho} \\
& \Longleftrightarrow x \Theta^{\prime} y \\
& \Longleftrightarrow[x]_{\Theta^{\prime}}=[y]_{\Theta^{\prime}} \\
& \Longleftrightarrow\left[\left([x]_{\Theta^{\prime}}, \alpha\right)\right]_{\rho}=\left[\left([y]_{\Theta^{\prime}}, \beta\right)\right]_{\rho} .
\end{aligned}
$$

This complete the proof.

Proposition 3.12. Let $G_{1}$ and $G_{2}$ be $\Gamma$-semihypergroups such that $G_{1} \cap$ $G_{2}=\emptyset$. Let $\Theta$ be a regular relation on $G_{1}\left[G_{2}\right]$. Then, the relation $\widehat{\Theta}$ is a regular relation on $\widehat{G_{1}}\left[\widehat{G_{2}}\right]$.

Proof. The proof is straightforward.

Proposition 3.13. Let $\Theta$ be a regular equivalence relation on semihypergroup $\widehat{G_{1}}\left[\widehat{G_{2}}\right]$. Then, $\Theta^{\prime}$ is a regular equivalence relation on $G_{1}\left[G_{2}\right]$.

Proof. Suppose that $x \Theta^{\prime} y$ and $z \in G_{1}\left[G_{2}\right]$. Therefore, $[(x, \Gamma)]_{\rho} \bar{\Theta}[(y, \Gamma)]_{\rho}$. Let $t_{1} \in x \otimes_{\alpha} z$. Then, $\left[\left(t_{1}, \alpha\right)\right]_{\rho} \in[(x, \alpha)]_{\rho} \circ[(z, \alpha)]_{\rho}$. From the regularity of $\Theta$, we have $[(x, \alpha)]_{\rho} \circ[(z, \alpha)]_{\rho} \bar{\Theta}[(y, \alpha)]_{\rho} \circ[(z, \alpha)]_{\rho}$. There exist $\left[\left(t_{2}, \alpha\right)\right]_{\rho} \in$ $[(y, \alpha)]_{\rho} \circ[(z, \alpha)]_{\rho}$ such that $\left[\left(t_{1}, \alpha\right)\right]_{\rho} \Theta\left[\left(t_{2}, \alpha\right)\right]_{\rho}$. We conclude that $t_{1} \Theta^{\prime} t_{2}$. So, we obtain $x \otimes_{\alpha} z \overline{\Theta^{\prime}} y \otimes_{\alpha} z$. 
Theorem 3.14. Let $G_{1}$ and $G_{2}$ be $\Gamma$-semihypergroups such that $G_{1} \cap G_{2}=\emptyset$ and let $\Theta$ be a regular relation on $G_{1}\left[G_{2}\right]$. Then, we have

$$
\left[G_{1} \widehat{\left[G_{2}\right]}: \Theta\right]=\left[\widehat{G_{1}}\left[\widehat{G_{2}}\right]: \widehat{\Theta}\right] .
$$

Definition 3.15. Let $G_{1}$ and $G_{2}$ be $\Gamma$-semihypergroups such that $G_{1} \cap G_{2}=$ $\emptyset$, let $\Theta_{1}$ and $\Theta_{2}$ be regular relations on $G_{1}\left[G_{2}\right]$, and let $\Theta_{1} \subseteq \Theta_{2}$. Then, the relation $\Theta_{1} / \Theta_{2}$ defined on $\left[G_{1}\left[G_{2}\right]: \Theta_{2}\right]$ define as follows:

$$
\left([x]_{\Theta_{2}},[y]_{\Theta_{2}}\right) \in \Theta_{1} / \Theta_{2} \Longleftrightarrow(x, y) \in \Theta_{1} .
$$

Proposition 3.16. Let $G_{1}$ and $G_{2}$ be $\Gamma$-semihypergroups such that $G_{1} \cap$ $G_{2}=\emptyset$, let $\Theta_{1}$ and $\Theta_{2}$ be regular relations on $G_{1}\left[G_{2}\right]$, and let $\Theta_{1} \subseteq \Theta_{2}$. Then,

$$
1: \widehat{\Theta_{1}} \subseteq \widehat{\Theta_{2}}
$$

2: The relation $\Theta_{1} / \Theta_{2}$ is regular,

$$
3: \widehat{\Theta_{1} / \Theta_{2}}=\widehat{\Theta_{1}} / \widehat{\Theta_{2}}
$$$$
4:\left[\left[G_{1}\left[G_{2}\right] \widehat{\left.: \Theta_{2}\right]}: \Theta_{1} / \Theta_{2}\right] \simeq\left[G_{1} \widehat{\left[G_{2}\right]:} \Theta_{1}\right] .\right.
$$

Proof. (1) : Suppose that $[(x, \alpha)]_{\rho} \widehat{\Theta_{1}}[(y, \beta)]_{\rho}$. So, $x \otimes_{\alpha} z \overline{\Theta_{1}} y \underset{\beta}{\otimes} z$, for all $z \in G_{1}\left[G_{2}\right]$. We conclude that $x \otimes_{\alpha} z \overline{\Theta_{2}} y \otimes_{\beta} z$, because $\Theta_{1} \subseteq \Theta_{2}$. This implies that $[(x, \alpha)]_{\rho} \widehat{\Theta_{2}}[(y, \beta)]_{\rho}$. Therefore, $\widehat{\Theta_{1}} \subseteq \widehat{\Theta_{2}}$. (2) : Let $[x]_{\Theta_{2}} \Theta_{1} / \Theta_{2}[y]_{\Theta_{2}}$ and let $[z]_{\Theta_{2}} \in\left[G_{1}\left[G_{2}\right]: \Theta_{2}\right]$. Therefore, $x \Theta_{1} y$. We obtain $x \otimes_{\alpha} z \overline{\Theta_{1}} y \otimes_{\alpha} z$, because $\Theta_{1}$ is regular. We conclude that $\left[x \otimes_{\alpha}\right.$ $z]_{\Theta_{2}} \overline{\Theta_{1} / \Theta_{2}}\left[\left(y \otimes_{\alpha} z\right)\right]_{\Theta_{2}}$. This implies that

$$
[x]_{\Theta_{2}} \underset{\alpha}{\oplus}[z]_{\Theta_{2}} \overline{\Theta_{1} / \Theta_{2}}[y]_{\Theta_{2}} \underset{\alpha}{\oplus}[z]_{\Theta_{2}} .
$$

So, $\Theta_{1} / \Theta_{2}$ is regular.

(3) : We prove the equation as follows:

$$
\begin{aligned}
{\left[\left([(x, \alpha)]_{\rho}\right)\right]_{\widehat{\Theta_{2}}} \widehat{\Theta_{1}} / \widehat{\Theta_{2}}\left[\left([(y, \beta)]_{\rho}\right)\right]_{\widehat{\Theta_{2}}} } & \Longleftrightarrow[(x, \alpha)]_{\rho} \widehat{\Theta_{1}}[(y, \beta)]_{\rho} \\
& \Longleftrightarrow x \otimes_{\alpha} z \overline{\Theta_{1}} y \otimes_{\beta} z \text { for all } z \in G_{1}\left[G_{2}\right] \\
& \Longleftrightarrow\left[x \otimes_{\alpha} z\right]_{\Theta_{2}} \overline{\Theta_{1} / \Theta_{2}}\left[y \otimes_{\beta} z\right]_{\Theta_{2}} \\
& \Longleftrightarrow\left[\left([(x, \alpha)]_{\rho}\right)\right]_{\widehat{\Theta_{2}}} \widehat{\Theta_{1} / \Theta_{2}}\left[\left([(y, \beta)]_{\rho}\right)\right]_{\widehat{\Theta_{2}}} .
\end{aligned}
$$


(4) : We define the relation $\Psi:\left[\left[G_{1}\left[G_{2}\right] \widehat{\left.: \Theta_{2}\right]}: \Theta_{1} / \Theta_{2}\right] \longrightarrow\left[G_{1} \widehat{\left[G_{2}\right]}: \Theta_{1}\right]\right.$ such that

$$
\Psi\left(\left[\left(\left[\left([x]_{\Theta_{2}}\right)\right]_{\Theta_{1} / \Theta_{2}}, \alpha\right)\right]_{\rho}\right)=\left[\left([x]_{\Theta_{1}}, \alpha\right)\right]_{\rho} .
$$

Now, we show that $\Psi$ is well-defined, one to one, and onto. Suppose that $\left[\left([x]_{\Theta_{1}}, \alpha\right)\right]_{\rho}=\left[\left([y]_{\Theta_{1}}, \beta\right)\right]_{\rho}$. Then, $[x]_{\Theta_{1}} \otimes_{\alpha}[z]_{\Theta_{1}}=[y]_{\Theta_{1}} \otimes_{\beta}[z]_{\Theta_{1}}$, for all $[z]_{\Theta_{1}} \in\left[G_{1}\left[G_{2}\right]: \Theta_{1}\right]$. Therefore $\left[x \otimes_{\alpha} z\right]_{\Theta_{1}}=[y \underset{\beta}{\otimes} z]_{\Theta_{1}}$. This implies that $(x \underset{\alpha}{\otimes} z) \overline{\Theta_{1}}(y \underset{\beta}{\otimes} z)$. We conclude that $(x \underset{\alpha}{\otimes} z) \overline{\Theta_{2}}(y \underset{\beta}{\otimes} z)$, because $\Theta_{1} \subseteq \Theta_{2}$. Therefore, $[(x \underset{\alpha}{\otimes} z)]_{\Theta_{2}}=[(y \underset{\beta}{\otimes} z)]_{\Theta_{2}}$. We obtain $[x]_{\Theta_{2}} \otimes_{\alpha}[z]_{\Theta_{2}}=[y]_{\Theta_{2}} \underset{\beta}{\otimes}[z]_{\Theta_{2}}$. Then,

$$
\left[\left(\left[\left([x]_{\Theta_{2}}\right)\right]_{\Theta_{1} / \Theta_{2}}, \alpha\right)\right]_{\rho}=\left[\left(\left[\left([y]_{\Theta_{2}}\right)\right]_{\Theta_{1} / \Theta_{2}}, \beta\right)\right]_{\rho} .
$$

So $\Psi$ is one to one. It's easy to see that $\Psi$ is onto. Suppose that $\left[\left(\left[\left([x]_{\Theta_{2}}\right)\right]_{\Theta_{1} / \Theta_{2}}, \alpha\right)\right]_{\rho}=$ $\left[\left(\left[\left([y]_{\Theta_{2}}\right)\right]_{\Theta_{1} / \Theta_{2}}, \beta\right)\right]_{\rho}$. Therefore,

$$
\left[\left([x]_{\Theta_{2}}\right)\right]_{\Theta_{1} / \Theta_{2}} \otimes_{\alpha}\left[\left([z]_{\Theta_{2}}\right)\right]_{\Theta_{1} / \Theta_{2}}=\left[\left([y]_{\Theta_{2}}\right)\right]_{\Theta_{1} / \Theta_{2}} \otimes_{\beta}\left[\left([z]_{\Theta_{2}}\right)\right]_{\Theta_{1} / \Theta_{2}}
$$

for all $\left[\left([z]_{\Theta_{2}}\right)\right]_{\Theta_{1} / \Theta_{2}} \in\left[\left[G_{1}\left[G_{2}\right]: \Theta_{2}\right]: \Theta_{1} / \Theta_{2}\right]$. We conclude that

$$
\left[\left([x]_{\Theta_{2}} \otimes_{\alpha}[z]_{\Theta_{2}}\right)\right]_{\Theta_{1} / \Theta_{2}}=\left[\left([y]_{\Theta_{2}} \otimes_{\beta}[z]_{\Theta_{2}}\right)\right]_{\Theta_{1} / \Theta_{2}} .
$$

Therefore, $\left[x \otimes_{\alpha} z\right]_{\Theta_{2}} \overline{\Theta_{1} / \Theta_{2}}[y \underset{\beta}{\otimes} z]_{\Theta_{2}}$. By the definition of $\Theta_{1} / \Theta_{2}$, we obtain $x \underset{\alpha}{z} \overline{\Theta_{1}} y \underset{\beta}{\otimes} z$. Therefore, $[x z]_{\Theta_{1}}=[y \underset{\beta}{\otimes} z]_{\Theta_{1}}$. This implies

$$
[x]_{\Theta_{1}} \otimes_{\alpha}[z]_{\Theta_{1}}=[y]_{\Theta_{1}} \underset{\beta}{\otimes}[z]_{\Theta_{1}} .
$$

So, $\left[\left([x]_{\Theta_{1}}, \alpha\right)\right]_{\rho}=\left[\left([y]_{\Theta_{1}}, \beta\right)\right]_{\rho}$ and $\Psi$ is well-defined.

Theorem 3.17. Let $G_{1}$ and $G_{2}$ be $\Gamma$-semihypergroups such that $G_{1} \cap G_{2}=\emptyset$ and let $\Theta_{1} \subseteq \Theta_{2}$ be regular relations on $G_{1}\left[G_{2}\right]$. Then,

$$
\overline{A p r}_{\Theta_{1} / \Theta_{2}}\left([A]_{\Theta_{2}}\right)=\left[\overline{A p r}_{\Theta_{1}}(A)\right]_{\Theta_{2}} .
$$

Proof. Suppose that $[x]_{\Theta_{2}} \in \overline{A p r}_{\Theta_{1} / \Theta_{2}}\left([A]_{\Theta_{2}}\right)$. Therefore $\left[\left([x]_{\Theta_{2}}\right)\right]_{\Theta_{1} / \Theta_{2}} \cap$ $[A]_{\Theta_{2}} \neq \emptyset$. There exist $[z]_{\Theta_{2}} \in\left[\left([x]_{\Theta_{2}}\right)\right]_{\Theta_{1} / \Theta_{2}} \cap[A]_{\Theta_{2}}$. Then $[z]_{\Theta_{2}} \in$ $\left[\left([x]_{\Theta_{2}}\right)\right]_{\Theta_{1} / \Theta_{2}}$ and $[z]_{\Theta_{2}} \in[A]_{\Theta_{2}}$. This implies that $[z]_{\Theta_{2}} \Theta_{1} / \Theta_{2}[x]_{\Theta_{2}}$. Then $z \Theta_{1} x$, by the definition of $\Theta_{1} / \Theta_{2}$. We have $z \in A$ and $z \in[x]_{\Theta_{1}}$ implies that $[x]_{\Theta_{1}} \cap A \neq \emptyset$. Therefore $x \in \overline{A p r}_{\Theta_{1}}(A)$. We conclude that 
$[x]_{\Theta_{2}} \in\left[\left(\overline{A p r}_{\Theta_{1}}(A)\right)\right]_{\Theta_{2}}$ and the inclusion $\overline{A p r}_{\Theta_{1} / \Theta_{2}}\left([A]_{\Theta_{2}}\right) \subseteq\left[\overline{A p r}_{\Theta_{1}}(A)\right]_{\Theta_{2}}$ obtained.

Conversely, suppose that $[x]_{\Theta_{2}} \in\left[\overline{A p r}_{\Theta_{1}}(A)\right]_{\Theta_{2}}$. Then $x \in \overline{A p r}_{\Theta_{1}}(A)$. This means that $[x]_{\Theta_{1}} \cap A \neq \emptyset$. There exist $z \in[x]_{\Theta_{1}} \cap A$. So $z \in A$ and $z \in[x]_{\Theta_{1}}$. Therefore $z \Theta_{1} x$ and $[z]_{\Theta_{2}} \in[A]_{\Theta_{2}}$. Then $[z]_{\Theta_{2}} \Theta_{1} / \Theta_{2}[x]_{\Theta_{2}}$. We conclude that $[z]_{\Theta_{2}} \in\left[\left([x]_{\Theta_{2}}\right)\right]_{\Theta_{1} / \Theta_{2}} \cap[A]_{\Theta_{2}}$. So, $\left[\left([x]_{\Theta_{2}}\right)\right]_{\Theta_{1} / \Theta_{2}} \cap[A]_{\Theta_{2}} \neq \emptyset$. This means that $[x]_{\Theta_{2}} \in \overline{A p r}_{\Theta_{1} / \Theta_{2}}\left([A]_{\Theta_{2}}\right)$. We obtain $\left[\overline{A p r}_{\Theta_{1}}(A)\right]_{\Theta_{2}} \subseteq$ $\overline{A p r}_{\Theta_{1} / \Theta_{2}}\left([A]_{\Theta_{2}}\right)$.

Theorem 3.18. Let $G_{1}$ and $G_{2}$ be $\Gamma$-semihypergroups such that $G_{1} \cap G_{2}=\emptyset$ and let $\Theta_{1} \subseteq \Theta_{2}$ be regular relations on $G_{1}\left[G_{2}\right]$. Then,

$$
\underline{A p r}_{\Theta_{1} / \Theta_{2}}\left([A]_{\Theta_{2}}\right)=\left[\underline{A p r}_{\Theta_{1}}(A)\right]_{\Theta_{2}} .
$$

Proof. Let $[x]_{\Theta_{2}} \in \underline{A p r}{\underline{\Theta_{1} / \Theta_{2}}}\left([A]_{\Theta_{2}}\right)$. Then $\left[\left([x]_{\Theta_{2}}\right)\right]_{\Theta_{1} / \Theta_{2}} \subseteq[A]_{\Theta_{2}}$. Suppose that $z \in[x]_{\Theta_{1}}$. This means that $z \Theta_{1} x$. Therefore $[z]_{\Theta_{2}} \Theta_{1} / \Theta_{2}[x]_{\Theta_{2}}$. So $[z]_{\Theta_{2}} \in\left[\left([x]_{\Theta_{2}}\right)\right]_{\Theta_{1} / \Theta_{2}} \subseteq[A]_{\Theta_{2}}$. We conclude that $[z]_{\Theta_{2}} \in[A]_{\Theta_{2}}$. Then $z \in A$ and we obtain $[x]_{\Theta_{1}} \subseteq A$. This means that $x \in \underline{A p r}{ }_{\Theta_{1}}(A)$. Hence $[x]_{\Theta_{2}} \in\left[\underline{\operatorname{Apr}} \Theta_{\Theta_{1}}(A)\right]_{\Theta_{2}}$.

Conversely, suppose that $[x]_{\Theta_{2}} \in\left[\left(\underline{A p r}_{\Theta_{1}}(A)\right)\right]_{\Theta_{2}}$. Then $x \in \underline{A p r} \underline{\Theta}_{1}(A)$. Therefore $[x]_{\Theta_{1}} \subseteq A$. Let $[z]_{\Theta_{2}} \in\left[\left([x]_{\Theta_{2}}\right)\right]_{\Theta_{1} / \Theta_{2}}$. So $[z]_{\Theta_{2}} \overline{\Theta_{1} / \Theta_{2}}[x]_{\Theta_{2}}$. This implies that $z \Theta_{1} x$. We have $z \in[x]_{\Theta_{1}} \subseteq A$. So $z \in A$ and $[z]_{\Theta_{2}} \in[A]_{\Theta_{2}}$. We conclude that $\left[\left([x]_{\Theta_{2}}\right)\right]_{\Theta_{1} / \Theta_{2}} \subseteq[A]_{\Theta_{2}}$. We obtain $[x]_{\Theta_{2}} \in \underline{A p r}{\underline{\Theta_{1} / \Theta_{2}}}\left([A]_{\Theta_{2}}\right)$.

Proposition 3.19. Suppose that $G_{1}$ and $G_{2}$ are $\Gamma$-semihypergroups. Then, 1) $\left(\mathcal{P}\left(\widehat{G_{1}}\left[\widehat{G_{2}}\right]\right)\right.$, o $)$ is a semihypergroup.

Proof. We know that $\left(\widehat{G_{1}}\left[\widehat{G_{2}}\right], \circ\right)$ is a semihypergroup. Suppose that $A, B, C \in$ $\mathcal{P}\left(\widehat{G_{1}}\left[\widehat{G_{2}}\right]\right)$. Then

$$
\begin{aligned}
(A \circ B) \circ C & =\bigcup_{[(x, \alpha)]_{\rho} \in A,[(y, \beta)]_{\rho} \in B,[(z, \gamma)]_{\rho} \in C}\left([(x, \alpha)]_{\rho} \circ[(y, \beta)]_{\rho}\right) \circ[(z, \gamma)]_{\rho} \\
& \left.=\bigcup_{[(x, \alpha)]_{\rho} \in A,[(y, \beta)]_{\rho} \in B,[(z, \gamma)]_{\rho} \in C}[(x, \alpha)]_{\rho} \circ\left([(y, \beta)]_{\rho}\right) \circ[(z, \gamma)]_{\rho}\right) \\
& =A \circ(B \circ C) .
\end{aligned}
$$


Proposition 3.20. Suppose that $G_{1}$ and $G_{2}$ are $\Gamma$-hyperideals such that $G_{1} \cap G_{2}=\emptyset$. If $(F, A)$ is a soft set over $\widehat{G_{1}}\left[\widehat{G_{2}}\right]$ such that $F$ is an onto function. Then, $\left(F^{\prime}, A^{\prime}\right)$ is a soft set over $G_{1}\left[G_{2}\right]$.

Proof. We define $F^{\prime}: A^{\prime} \longrightarrow G_{1}\left[G_{2}\right]$ as follows:

$$
F^{\prime}(x)=\left(F\left([(x, \Gamma)]_{\rho}\right)\right)^{\prime} \quad \text { forall } x \in A^{\prime} .
$$

We show that $F^{\prime}$ is well-defined. Let $x=y$ for $x, y \in A^{\prime}$. Then, $[(x, \Gamma)]_{\rho}=$ $[(y, \Gamma)]_{\rho}$. From the assumption, we conclude that $F\left([(x, \Gamma)]_{\rho}\right)=F\left([(y, \Gamma)]_{\rho}\right)$, because $F$ is well-defined. We obtain

$$
\left(F\left([(x, \Gamma)]_{\rho}\right)\right)^{\prime}=\left(F\left([(y, \Gamma)]_{\rho}\right)\right)^{\prime} .
$$

This implies that $F^{\prime}(x)=F^{\prime}(y)$ and $F^{\prime}$ is well-defined.

Definition 3.21. Suppose that $(F, A)$ is a soft set over $\widehat{G_{1}}\left[\widehat{G_{2}}\right]$. We denote the soft set $\left(F^{\prime}, A^{\prime}\right)$ by $(F, A)^{\prime}$ and we define

$$
\operatorname{supp}\left((F, A)^{\prime}\right)=\left\{x \in A^{\prime} \mid F^{\prime}(x) \neq \emptyset\right\} .
$$

Proposition 3.22. Suppose that $A$ is a right hyperideal of semihypergroup E. Therefore, we have

$$
x \in \operatorname{supp}\left((F, A)^{\prime}\right) \Longleftrightarrow[(x, \Gamma)]_{\rho} \subseteq \operatorname{supp}(F, A) .
$$

Proof. Let $x \in \operatorname{supp}(F, A)^{\prime}$. Then $x \in A^{\prime}$ and $F^{\prime}(x) \neq \emptyset$. There exist $\alpha \in \Gamma$ such that $[(x, \alpha)]_{\rho} \in A$. We have $F^{\prime}(x)=F\left([(x, \Gamma)]_{\rho}\right)^{\prime}$. We know that $F^{\prime}(x) \neq \emptyset$. Therefore $F\left([(x, \Gamma)]_{\rho}\right)^{\prime} \neq \emptyset$. Then, there exist $y \in F\left([(x, \Gamma)]_{\rho}\right)^{\prime}$. There exist $\beta \in \Gamma$ such that $[(y, \beta)]_{\rho} \in F\left([(x, \Gamma)]_{\rho}\right)$. So $F\left([(x, \Gamma)]_{\rho}\right) \neq \emptyset$. We conclude that $[(x, \Gamma)]_{\rho} \subseteq \operatorname{supp}(F, A)$.

Conversely, let $[(x, \Gamma)]_{\rho} \subseteq \operatorname{supp}(F, A)$. Then for every $\alpha \in \Gamma,[(x, \alpha)]_{\rho} \in$ $\operatorname{supp}(F, A)$. This implies that $[(x, \alpha)]_{\rho} \in A$ and $F\left([(x, \alpha)]_{\rho}\right) \neq \emptyset$. Then $x \in A^{\prime}$ and $F\left([(x, \alpha)]_{\rho}\right)^{\prime} \neq \emptyset$. We conclude that $x \in A^{\prime}$ and $F\left([(x, \Gamma)]_{\rho}\right)^{\prime} \neq \emptyset$. We know that $F^{\prime}(x)=F\left([(x, \Gamma)]_{\rho}\right)^{\prime}$. So $F^{\prime}(x) \neq \emptyset$ and $x \in A^{\prime}$ which implies that $x \in \operatorname{supp}\left((F, A)^{\prime}\right)$.

Theorem 3.23. Let $A$ be a right hyperideal over semihypergroup E. Then,

$$
(\operatorname{supp}(F, A))^{\prime}=\operatorname{supp}\left(F^{\prime}, A^{\prime}\right) .
$$


Proof. Suppose that $x \in(\operatorname{supp}(F, A))^{\prime}$. There exist $\alpha \in \Gamma$ such that $[(x, \alpha)]_{\rho} \in \operatorname{supp}(F, A)$. Then $[(x, \alpha)]_{\rho} \in A$ and $F\left([(x, \alpha)]_{\rho}\right) \neq \emptyset$. So $x \in A^{\prime}$ and $F\left([(x, \alpha)]_{\rho}\right)^{\prime} \neq \emptyset$. We conclude that $x \in A^{\prime}$ and $F^{\prime}(x) \neq \emptyset$. This implies that $x \in \operatorname{supp}\left(F^{\prime}, A^{\prime}\right)$. We obtain $(\operatorname{supp}(F, A))^{\prime} \subseteq \operatorname{supp}\left(F^{\prime}, A^{\prime}\right)$.

Conversely, let $x \in \operatorname{supp}\left(F^{\prime}, A^{\prime}\right)$. Therefore $x \in A^{\prime}$ and $F^{\prime}(x) \neq \emptyset$. We conclude that $F\left([(x, \Gamma)]_{\rho}\right) \neq \emptyset$ and for every $\alpha \in \Gamma$, we have $[(x, \Gamma)]_{\rho} \subseteq A$. We obtain $[(x, \Gamma)]_{\rho} \subseteq \operatorname{supp}(F, A)$. So $x \in(\operatorname{supp}(F, A))^{\prime}$.

Proposition 3.24. Suppose that $A$ is a semihypergroup and $(F, A)$ is a soft set over $\widehat{G_{1}}\left[\widehat{G_{2}}\right]$. Then,

$$
\left(F\left([(x, \Gamma)]_{\rho}\right)\right)^{\prime} \subseteq F^{\prime}(x) .
$$

Proof. Let $y \in\left(F\left([(x, \Gamma)]_{\rho}\right)\right)^{\prime}$. There exist $\alpha \in \Gamma$ such that $[(y, \alpha)]_{\rho} \in$ $F\left([(x, \Gamma)]_{\rho}\right)$. There exist $\beta \in \Gamma$ such that $[(y, \alpha)]_{\rho} \in F\left([(x, \beta)]_{\rho}\right) \subseteq\left[\left(F^{\prime}(x), \beta\right)\right]_{\rho}$. So, There exist $t \in F^{\prime}(x)$ such that $[(y, \alpha)]_{\rho}=[(t, \beta)]_{\rho}$. We have $y \otimes_{\alpha} z=$ $t \otimes_{\beta} z$, for every $z \in G_{1}\left[G_{2}\right]$. We set $z=e_{\alpha}$. we obtain $y \otimes_{\alpha} e_{\alpha}=t \underset{\beta}{\otimes_{\alpha}} e_{\alpha}$. This implies that

$$
y \in t \underset{\beta}{\otimes} e_{\alpha} \subseteq F^{\prime}(x) \underset{\beta}{\otimes} G_{1}\left[G_{2}\right] \subseteq F^{\prime}(x) .
$$

We conclude that $y \in F^{\prime}(x)$ and $\left(F\left([(x, \Gamma)]_{\rho}\right)\right)^{\prime} \subseteq F^{\prime}(x)$.

There is a connection between subsemihypergroups of semihypergroup $\widehat{G_{1}}\left[\widehat{G_{2}}\right]$ and $\Gamma$-subsemihypergroups of associated $\Gamma$-hypergroupoid $G_{1}\left[G_{2}\right]$ as follows:

Proposition 3.25. Let $A$ be a non-empty subset of $\widehat{G_{1}}\left[\widehat{G_{2}}\right]$ and let $A$ be a subsemihypergroup of $\widehat{G_{1}}\left[\widehat{G_{2}}\right]$. Therefore, $A^{\prime}$ is a $\Gamma$-subsemihypergroup of $G_{1}\left[G_{2}\right]$.

Proof. Let $x, y \in A^{\prime}$. There exist $\alpha, \beta \in \Gamma$ such that $[(x, \alpha)]_{\rho},[(y, \beta)]_{\rho} \in A$. Then, by the assumption, we have $[(x, \alpha)]_{\rho} \circ[(y, \beta)]_{\rho} \subseteq A$. This implies that $\left[\left(x \otimes_{\alpha} y, \beta\right)\right]_{\rho} \subseteq A$. This means that $x \otimes_{\alpha} y \subseteq A^{\prime}$.

Definition 3.26. We say that $\left(\widehat{G_{1}}\left[\widehat{G_{2}}\right], \Theta\right)$ is a Pawlak approximation space, where $\widehat{G_{1}}\left[\widehat{G_{2}}\right]$ is a semihypergroup and $\Theta$ is an equivalence relation over $\widehat{G_{1}}\left[\widehat{G_{2}}\right]$.

Definition 3.27. Let $\left(\widehat{G_{1}}\left[\widehat{G_{2}}\right], \Theta\right)$ be a Pawlak approximation space and let $G=(F, A)$ be a soft set over $\widehat{G_{1}}\left[\widehat{G_{2}}\right]$. Then, $\underline{U}(\Theta, G)$ is called a lower 
rough soft semihypergroup w.r.t $\Theta$ of $\widehat{G_{1}}\left[\widehat{G_{2}}\right]$ if $\underline{A p r_{\Theta}} F\left([(x, \alpha)]_{\rho}\right)$ is a subsemihypergroup of $\widehat{G_{1}}\left[\widehat{G_{2}}\right]$, for every $[(x, \alpha)]_{\rho} \in A$. Also, we can define lower rough soft hyperideal and lower rough soft prime hyperideal in this way. Moreover, $G$ is called a rough soft semihypergroup w.r.t $\Theta$ of $\widehat{G_{1}}\left[\widehat{G_{2}}\right]$ if $\operatorname{Apr}_{\Theta} F\left([(x, \alpha)]_{\rho}\right)$ and $\overline{A p r}_{\Theta} F\left([(x, \alpha)]_{\rho}\right)$ are subsemihypergroups of $\widehat{G_{1}}\left[\widehat{G_{2}}\right]$, for all $[(x, \alpha)]_{\rho} \in A$.

ExAmple 12. In Example $9,\left(\widehat{G_{1}}\left[\widehat{G_{2}}\right], \widehat{\Theta}\right)$ is a Pawlak approximation space. Suppose that $G=(F, A)$ be a soft set over $\widehat{G_{1}}\left[\widehat{G_{2}}\right]$, where $A=\left\{[(m, \alpha)]_{\rho},[(n, \alpha)]_{\rho}\right\}$ and

$$
F\left([(m, \alpha)]_{\rho}\right)=\widehat{G_{2}}, \quad F\left([(n, \alpha)]_{\rho}\right)=\left\{[(a, \alpha)]_{\rho},[(b, \beta)]_{\rho}\right\} .
$$

Therefore,

$$
\begin{aligned}
& \underline{F}_{\widehat{\Theta}}\left([(m, \alpha)]_{\rho}\right)=\bar{F}_{\widehat{\Theta}}\left([(m, \alpha)]_{\rho}\right)=\widehat{G_{2}}, \\
& \underline{F}_{\widehat{\Theta}}\left([(n, \alpha)]_{\rho}\right)=\emptyset, \bar{F}_{\widehat{\Theta}}\left([(n, \alpha)]_{\rho}\right)=\widehat{G_{1}} .
\end{aligned}
$$

Thus, $G=(F, A)$ is a rough soft semihypergroup over $\widehat{G_{1}}\left[\widehat{G_{2}}\right]$.

Proposition 3.28. Suppose that $\widehat{G_{1}}\left[\widehat{G_{2}}\right]$ is a semihypergroup with unit and $(F, A)$ is a soft right hyperideal over $\widehat{G_{1}}\left[\widehat{G_{2}}\right]$ such that $A$ is a semihypergroup. Then,

$$
\left(F\left([(x, \Gamma)]_{\rho}\right)\right)^{\prime}=F^{\prime}(x) .
$$

Proof. Let $y \in F^{\prime}(x)$. Then, $[(y, \Gamma)]_{\rho} \in\left[\left(F^{\prime}(x), \Gamma\right)\right]_{\rho}=F\left([(x, \Gamma)]_{\rho}\right)$. So $[(y, \Gamma)]_{\rho} \subseteq F\left([(x, \Gamma)]_{\rho}\right)$. This implies that $y \in\left(F\left([(x, \Gamma)]_{\rho}\right)\right)^{\prime}$. We obtain $F^{\prime}(x) \subseteq\left(F\left([(x, \Gamma)]_{\rho}\right)\right)^{\prime}$. Now, let $y \in\left(F\left([(x, \Gamma)]_{\rho}\right)\right)^{\prime}$. There exist $\alpha \in \Gamma$ such that $[(y, \alpha)]_{\rho} \in F\left([(x, \Gamma)]_{\rho}\right)=\left[\left(F^{\prime}(x), \Gamma\right)\right]_{\rho}$. We conclude that $y \in F^{\prime}(x)$ and $\left(F\left([(x, \Gamma)]_{\rho}\right)\right)^{\prime} \subseteq F^{\prime}(x)$.

\section{Relation between decision making algorithm of rough soft semihypergroups and associated rough soft sets}

In this section, first we obtain the decision making algorithm of rough soft semihypergroups $\widehat{G_{1}}\left[\widehat{G_{2}}\right]$, then we find a relation between the decision making algorithm of this rough soft semihypergroup and its associated rough soft set.

Decision making method I: Let $\widehat{G_{1}}\left[\widehat{G_{2}}\right]$ be a semihypergroup and $E$ be a set of related parameters. Then, $G=(F, A)$ is called original description 
soft set over $\widehat{G_{1}}\left[\widehat{G_{2}}\right]$, where $A=\left\{\left[\left(e_{1}, \alpha_{1}\right)\right]_{\rho},\left[\left(e_{2}, \alpha_{2}\right)\right]_{\rho}, \cdots,\left[\left(e_{m}, \alpha_{m}\right)\right]_{\rho}\right\} \subseteq$ $E$, let $\Theta$ be an equivalence relation of $\widehat{G_{1}}\left[\widehat{G_{2}}\right]$ and let $\left(\widehat{G_{1}}\left[\widehat{G_{2}}\right], \Theta\right)$ be a Pawlak approximation space. Then, we present the decision algorithm for rough soft sets on semihypergroups as follows:

step 1: Input the original description semihypergroup $\widehat{G_{1}}\left[\widehat{G_{2}}\right]$, soft set $G$ and Pawlak approximation space $\left(\widehat{G_{1}}\left[\widehat{G_{2}}\right], \Theta\right)$.

step 2: Compute the lower and upper rough soft approximations $\underline{\operatorname{Apr}} \underline{\Theta}_{\Theta}(G)$ and $\overline{A p r}_{\Theta}(G)$ on $G$, respectively.

step 3: Compute the different values of $\left\|F\left(\left[e_{i}, \alpha_{i}\right]_{\rho}\right)\right\|$, where

$$
\left\|F\left(\left[e_{i}, \alpha_{i}\right]_{\rho}\right)\right\|=\frac{\left|\overline{A p r}_{\Theta}\left(F\left(\left[e_{i}, \alpha_{i}\right]_{\rho}\right)\right)\right|-\left|\overline{A p r}_{\Theta}\left(F\left(\left[e_{i}, \alpha_{i}\right]_{\rho}\right)\right)\right|}{\left|F\left(\left[e_{i}, \alpha_{i}\right]_{\rho}\right)\right|} .
$$

step 4: Find the minimum value $\left\|F\left(\left[e_{k}, \alpha_{k}\right]_{\rho}\right)\right\|$ of $\left\|F\left(\left[e_{i}, \alpha_{i}\right]_{\rho}\right)\right\|$, where

$$
\left\|F\left(\left[e_{k}, \alpha_{k}\right]_{\rho}\right)\right\|=\min _{1 \leq i \leq n}\left\{\left\|F\left(\left[e_{i}, \alpha_{i}\right]_{\rho}\right)\right\|\right\} .
$$

step 5: The decision is $F\left(\left[e_{k}, \alpha_{k}\right]_{\rho}\right)$.

Example 13. Consider Example 12, we define a soft set $G=(F, A)$ over $\widehat{G_{1}}\left[\widehat{G_{2}}\right]$, where $A=\left\{[(m, \alpha)]_{\rho},[(n, \alpha)]_{\rho},[(l, \alpha)]_{\rho}\right\}$ such that

$$
\begin{aligned}
& F\left([(m, \alpha)]_{\rho}\right)=\left\{[(a, \alpha)]_{\rho},[(a, \beta)]_{\rho},[(c, \alpha)]_{\rho},[(c, \beta)]_{\rho},\right.
\end{aligned}
$$

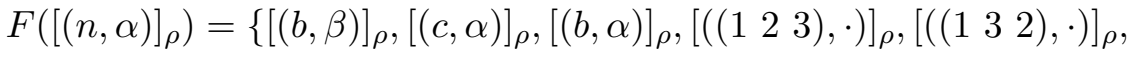

$$
\begin{aligned}
& F\left([(l, \alpha)]_{\rho}\right)=\left\{\left[\left(\left(\begin{array}{lll}
1 & 3 & 2
\end{array}\right), \cdot\right)\right]_{\rho},[(a, \alpha)]_{\rho}\right\} .
\end{aligned}
$$

We obtain

$$
\begin{aligned}
& \underline{A p r}_{\widehat{\Theta}} F\left([(m, \alpha)]_{\rho}\right)=\emptyset, \quad \overline{A p r}_{\widehat{\Theta}} F\left([(m, \alpha)]_{\rho}\right)=\widehat{G}_{1}, \\
& \underline{A p r}_{\widehat{\Theta}} F\left([(n, \alpha)]_{\rho}\right)=\left\{\left[\left(\left(\begin{array}{lll}
1 & 2 & 3
\end{array}\right), \cdot\right)\right]_{\rho},\left[\left(\left(\begin{array}{lll}
1 & 3 & 2
\end{array}\right), \cdot\right)\right]_{\rho}\right\}, \overline{A p r}_{\widehat{\Theta}} F\left([(n, \alpha)]_{\rho}\right) . \\
& =\widehat{G_{1}} \cup\left\{\left[\left(\left(\begin{array}{lll}
1 & 2 & 3
\end{array}\right), \cdot\right)\right]_{\rho},\left[\left(\left(\begin{array}{lll}
1 & 3 & 2
\end{array}\right), \cdot\right)\right]_{\rho}\right\}, \\
& \operatorname{Apr}_{\widehat{\Theta}} F\left([(l, \alpha)]_{\rho}\right)=\left\{[((132), \cdot)]_{\rho}\right\}, \quad \overline{A p r}_{\widehat{\Theta}} F\left([(l, \alpha)]_{\rho}\right)=\widehat{G_{1}} \cup\left\{[((132), \cdot)]_{\rho}\right\} .
\end{aligned}
$$

Then,

$$
\begin{gathered}
\left\|F\left([(m, \alpha)]_{\rho}\right)\right\|=\frac{6-0}{4}=1.5 \\
\left\|F\left([(n, \alpha)]_{\rho}\right)\right\|=\frac{8-2}{5}=1.2 \\
\left\|F\left([(l, \alpha)]_{\rho}\right)\right\|=\frac{7-1}{2}=3 .
\end{gathered}
$$

The decision is $F\left([(n, \alpha)]_{\rho}\right)$. 
Theorem 4.1. Let $(F, A)$ be a rough soft hyperideal over $\widehat{G_{1}}\left[\widehat{G_{2}}\right]$ w.r.t $\Theta$ and $\Theta$ be a regular relation. Then, for every $\left[\left(e_{i}, \alpha_{i}\right)\right]_{\rho} \in A$ such that $F\left(\left[\left(e_{i}, \alpha_{i}\right)\right]_{\rho}\right) \neq \emptyset$, we have

$$
\left(\underline{A p r}_{\Theta} F\left(\left[\left(e_{i}, \alpha_{i}\right)\right]_{\rho}\right)\right)^{\prime} \subseteq \underline{A p r}_{\Theta^{\prime}}\left(F\left(\left[\left(e_{i}, \alpha_{i}\right)\right]_{\rho}\right)\right)^{\prime} .
$$

Proof. By the assumption $F\left(\left[\left(e_{i}, \alpha_{i}\right)\right]_{\rho}\right) \neq \emptyset$ is a hyperideal over $\widehat{G_{1}}\left[\widehat{G_{2}}\right]$ w.r.t $\Theta$, for every $\left[\left(e_{i}, \alpha_{i}\right)\right]_{\rho} \in A$. Then, By Theorem 3.6, the proof is complete.

Theorem 4.2. Let $(F, A)$ be a non-null rough soft set over $\widehat{G_{1}}\left[\widehat{G_{2}}\right]$ w.r.t $\Theta$. Then, for every $\left[\left(e_{i}, \alpha_{i}\right)\right]_{\rho} \in A$ such that $F\left(\left[\left(e_{i}, \alpha_{i}\right)\right]_{\rho}\right) \neq \emptyset$, we have

$$
\left(\overline{\operatorname{Apr}}_{\Theta} F\left(\left[\left(e_{i}, \alpha_{i}\right)\right]_{\rho}\right)\right)^{\prime}=\overline{\operatorname{Apr}}_{\Theta^{\prime}}\left(F\left(\left[\left(e_{i}, \alpha_{i}\right)\right]_{\rho}\right)\right)^{\prime} .
$$

Proof. By Theorem 3.8, we obtain the equation.

Proposition 4.3. Suppose that $\left(\widehat{G_{1}}\left[\widehat{G_{2}}\right], \Theta\right)$ is a Pawlak approximation space. Then, $\left(\widehat{G_{1}}\left[\widehat{G_{2}}\right], \Theta\right)^{\prime}$ is also a Pawlak approximation space.

Proof. By the assumption, $\Theta$ is a regular relation on semihypergroup $\widehat{G_{1}}\left[\widehat{G_{2}}\right]$ and by Proposition $3.13, \Theta^{\prime}$ is a regular relation on $G_{1}\left[G_{2}\right]$. So, $\left(G_{1}\left[G_{2}\right], \Theta^{\prime}\right)$ is a Pawlak approximation space.

Proposition 4.4. Let $\Theta$ be a regular relation on $\widehat{G_{1}}\left[\widehat{G_{2}}\right]$. Then,

$$
\underline{A p r}_{\Theta} F\left(\left[\left(e_{i}, \Gamma\right)\right]_{\rho}\right) \subseteq\left[\left(\underline{A p r}_{\Theta} F^{\prime}\left(e_{i}\right), \Gamma\right)\right]_{\rho} .
$$

Proof. First, we prove that $F\left(\left[\left(e_{i}, \Gamma\right)\right]_{\rho}\right) \subseteq\left[\left(F^{\prime}\left(e_{i}\right), \Gamma\right)\right]_{\rho}$. Let $[(x, \alpha)]_{\rho} \in$ $F\left(\left[\left(e_{i}, \Gamma\right)\right]_{\rho}\right)$. Then, $x \in F\left(\left[\left(e_{i}, \Gamma\right)\right]_{\rho}\right)^{\prime} \subseteq F^{\prime}\left(e_{i}\right)$. Thus, $x \in F^{\prime}\left(e_{i}\right)$. This implies that $[(x, \alpha)]_{\rho} \in\left[\left(F^{\prime}\left(e_{i}\right), \Gamma\right)\right]_{\rho}$. We conclude that $F\left(\left[\left(e_{i}, \Gamma\right)\right]_{\rho}\right) \subseteq$ $\left[\left(F^{\prime}\left(e_{i}\right), \Gamma\right)\right]_{\rho}$.

Now, let $[(x, \alpha)]_{\rho} \in \underline{A p r}_{\Theta} F\left(\left[\left(e_{i}, \Gamma\right)\right]_{\rho}\right)$. Then, $\left[\left([(x, \alpha)]_{\rho}\right)\right]_{\Theta} \subseteq F\left(\left[\left(e_{i}, \Gamma\right)\right]_{\rho}\right)$. Also, $t \in[x]_{\Theta^{\prime}}$, implies that $t \Theta^{\prime} x$ and $[(t, \Gamma)]_{\rho} \bar{\Theta}[(x, \Gamma)]_{\rho}$. So, for $\alpha \in \Gamma$, there exists $\beta \in \Gamma$ such that $[(t, \beta)]_{\rho} \Theta[(x, \alpha)]_{\rho}$. We conclude that $[(t, \beta)]_{\rho} \in$ $F\left(\left[\left(e_{i}, \Gamma\right)\right]_{\rho}\right) \subseteq\left[\left(F^{\prime}\left(e_{i}\right), \Gamma\right)\right]_{\rho}$. So, $t \in F^{\prime}\left(e_{i}\right)$. We obtain $[x]_{\Theta^{\prime}} \subseteq F^{\prime}\left(e_{i}\right)$. So, $x \in \underline{A p r}_{\Theta^{\prime}} F^{\prime}\left(e_{i}\right)$ and $[(x, \alpha)]_{\rho} \in\left[\left(\underline{A p r}_{\Theta^{\prime}} F^{\prime}\left(e_{i}\right), \Gamma\right)\right]_{\rho}$. Therefore, $\underline{A p r}_{\Theta} F\left(\left[\left(e_{i}, \Gamma\right)\right]_{\rho}\right) \subseteq\left[\left(\underline{A p r}_{\Theta^{\prime}} F^{\prime}\left(e_{i}\right), \Gamma\right)\right]_{\rho}$.

Theorem 4.5. Let $A$ and $\widehat{G_{1}}\left[\widehat{G_{2}}\right]$ be semihypergroups with unit and $(F, A)$ be a soft right hyperideal over $\widehat{G_{1}}\left[\widehat{G_{2}}\right]$ and $\Theta$ be a regular relation on $\widehat{G_{1}}\left[\widehat{G_{2}}\right]$. Then, for every $e_{i} \in A^{\prime}$, we have

$$
\underline{A p r}_{\Theta^{\prime}}\left(F\left(\left[\left(e_{i}, \Gamma\right)\right]_{\rho}\right)\right)^{\prime}=\underline{A p r}_{\Theta^{\prime}} F^{\prime}\left(e_{i}\right) .
$$


Proof. The proof obtained by Proposition 3.28.

Theorem 4.6. Let $A$ and $\widehat{G_{1}}\left[\widehat{G_{2}}\right]$ be semihypergroups with unit such that $(F, A)$ be a soft right hyperideal over $\widehat{G_{1}}\left[\widehat{G_{2}}\right]$ and $\Theta$ be a regular relation on $\widehat{G_{1}}\left[\widehat{G_{2}}\right]$. Then, for every $e_{i} \in A^{\prime}$, we have

$$
\overline{A p r}_{\Theta^{\prime}}\left(F\left(\left[\left(e_{i}, \Gamma\right)\right]_{\rho}\right)\right)^{\prime}=\overline{A p r}_{\Theta^{\prime}} F^{\prime}\left(e_{i}\right) .
$$

Proof. The proof obtained by Proposition 3.28.

Now, we obtain the decision making algorithm of associated rough soft set as follows:

step 1: Input associated set $\left(G_{1}\left[G_{2}\right], \otimes_{\alpha}\right)$, soft set $G^{\prime}=(F, A)^{\prime}$, Pawlak approximation space $\left(G_{1}\left[G_{2}\right], \Theta^{\prime}\right)$ where $\Theta^{\prime}$ is a regular relation of $G_{1}\left[G_{2}\right]$. step 2: For all $\left[\left(e_{i}, \alpha_{i}\right)\right]_{\rho} \in A$, we conclude that $e_{i} \in A^{\prime}$. Thus, we obtain the lower and upper rough soft approximations $\operatorname{Apr}_{\Theta^{\prime}}\left(F^{\prime}\left(e_{i}\right)\right)$ and $\overline{A p r}_{\Theta^{\prime}}\left(F^{\prime}\left(e_{i}\right)\right)$.

step 3: Compute the different values of $\left\|F^{\prime}\left(e_{i}\right)\right\|$ as follows:

$$
\begin{aligned}
\left\|F^{\prime}\left(e_{i}\right)\right\| & =\frac{\left|\overline{A p r}_{\Theta^{\prime}}\left(F^{\prime}\left(e_{i}\right)\right)\right|-\left|\underline{A p r}_{\Theta^{\prime}}\left(F^{\prime}\left(e_{i}\right)\right)\right|}{\left|F^{\prime}\left(e_{i}\right)\right|} \\
& \leq \frac{\left|\overline{A p r}_{\Theta^{\prime}}\left(F\left(\left[\left(e_{i}, \Gamma\right)\right]_{\rho}\right)\right)^{\prime}\right|-\left|\operatorname{Apr}_{\Theta^{\prime}}\left(F\left(\left[\left(e_{i}, \Gamma\right)\right]_{\rho}\right)\right)^{\prime}\right|}{\left|F^{\prime}\left(e_{i}\right)\right|} \\
& =\frac{\left|\left(\overline{A p r}_{\Theta} F\left(\left[\left(e_{i}, \Gamma\right)\right]_{\rho}\right)\right)^{\prime}\right|-\left|\left(\overline{A p r}_{\Theta} F\left(\left[\left(e_{i}, \Gamma\right)\right]_{\rho}\right)\right)^{\prime}\right|}{\left(F\left(\left[\left(e_{i}, \Gamma\right)\right]_{\rho}\right)\right)^{\prime}} \\
& =\left\|\left(F\left(\left[\left(e_{i}, \Gamma\right)\right]_{\rho}\right)\right)^{\prime}\right\| .
\end{aligned}
$$

step 4: Find the minimum value of $\left\|F^{\prime}\left(e_{k}\right)\right\|$, where

$$
\begin{aligned}
\left\|F^{\prime}\left(e_{k}\right)\right\| & =\min _{1 \leq i \leq n}\left\|F^{\prime}\left(e_{i}\right)\right\| \\
& =\min _{1 \leq i \leq n}\left\|\left(F\left(\left[\left(e_{i}, \Gamma\right)\right]_{\rho}\right)\right)^{\prime}\right\| \\
& =\left\|\left(F\left(\left[\left(e_{k}, \Gamma\right)\right]_{\rho}\right)\right)^{\prime}\right\| .
\end{aligned}
$$

step 5: The decision is $F^{\prime}\left(e_{k}\right)$

Example 14. Consider examples 12 and 13. We have $G^{\prime}=(F, A)^{\prime}$ such that $A^{\prime}=\{m, n, l\}$ and we obtain

$$
F^{\prime}(m)=\{a, c\}, \quad F^{\prime}(n)=\left\{b, c,\left(\begin{array}{lll}
1 & 2 & 3
\end{array}\right),\left(\begin{array}{lll}
1 & 3 & 2
\end{array}\right)\right\}, \quad F^{\prime}(l)=\left\{\left(\begin{array}{lll}
1 & 3 & 2
\end{array}\right), a\right\} .
$$


Also, $(\widehat{\Theta})^{\prime}$ is a regular relation on $G_{1}\left[G_{2}\right]$. So, we have

$$
[a]_{(\widehat{\Theta})^{\prime}}=[b]_{(\widehat{\Theta})^{\prime}}=[c]_{(\widehat{\Theta})^{\prime}}=G_{1}
$$

$$
\left.\left[\left(\begin{array}{lll}
1 & 2 & 3
\end{array}\right)\right]_{(\widehat{\Theta})^{\prime}}=\left\{\left(\begin{array}{lll}
1 & 2 & 3
\end{array}\right)\right\},\left[\left(\begin{array}{lll}
1 & 3 & 2
\end{array}\right)\right]_{(\widehat{\Theta})^{\prime}}=\left\{\begin{array}{lll}
1 & 3 & 2
\end{array}\right)\right\},\left[\left(\begin{array}{l}
1
\end{array}\right]_{(\widehat{\Theta})^{\prime}}=\{(1)\} .\right.
$$

Now, we obtain the lower and upper rough soft approximations as follows:

$$
\begin{gathered}
\underline{A p r}_{(\widehat{\Theta})^{\prime}} F^{\prime}(m)=\emptyset, \quad \overline{A p r}_{(\widehat{\Theta})^{\prime}} F^{\prime}(m)=G_{1} . \\
\underline{A p r}(\widehat{\Theta})^{\prime} F^{\prime}(n)=\left\{\left(\begin{array}{lll}
1 & 2 & 3
\end{array}\right),\left(\begin{array}{lll}
1 & 3 & 2
\end{array}\right), \quad \overline{A p r}_{(\widehat{\Theta})^{\prime}} F^{\prime}(n)=G_{1} \cup\left\{\left(\begin{array}{lll}
1 & 2 & 3
\end{array}\right),\left(\begin{array}{lll}
1 & 3 & 2
\end{array}\right)\right\} .\right. \\
\underline{A p r}(\widehat{\Theta})^{\prime} F^{\prime}(l)=\left\{\left(\begin{array}{lll}
1 & 3 & 2
\end{array}\right),, \quad \overline{A p r}_{(\widehat{\Theta})^{\prime}} F^{\prime}(l)=G_{1} \cup\left\{\begin{array}{lll}
1 & 3 & 2
\end{array}\right)\right\} .
\end{gathered}
$$

We compute the difference values as follows:

$$
\begin{gathered}
\left\|F^{\prime}(m)\right\|=\frac{3-0}{2}=1.50 \\
\left\|F^{\prime}(n)\right\|=\frac{5-2}{4}=0.75 \\
\left\|F^{\prime}(l)\right\|=\frac{4-1}{2}=1.50
\end{gathered}
$$

The decision is $F^{\prime}(n)$. Moreover, in the Example 13, we conclude that the appropriate parameter is $[(n, \alpha)]_{\rho}$.

\section{Conclusions}

The concept of rough soft sets introduced by Feng [16] by combining rough sets and soft sets. Also, rough soft sets to algebraic structureshemirings investigated by Zhan [51]. They gave some characterizations of rough soft hemirings. In the present paper, we put forward a novel rough soft algebraic hyperstructure extension semihypergroups induced by operators. Hence, we try to investigated relation between decision making algorithm of rough soft semihypergroups and associated rough soft sets. The concept of decision making has been found very important in the imprecise environment. Hence, we try to put decision making approaches based on rough soft extension semi hypergroups induced by operators and motivation for decision algorithm extensions semihypergroups induced by operators is to found which is the best parameter of a given soft set. We hope it would be served as a foundation of rough soft set theory and other decision making methods in different areas, such as theoretical computer sciences, information sciences and intelligent systems, and so on. 


\section{Conflict of interest}

The authors declare that they have no conflict of interest.

\section{Ethical approval}

This article does not contain any studies with human participants performed by any of the authors.

\section{Informed consent}

Informed consent was obtained from all individual participants included in the study.

\section{Authorship contributions}

All authors contributed to the study conception and design. Material preparation, data collection and analysis were performed by Noreh Rakhsh Khorshid and Sohrab OstadhadiDehkordi. The first draft of the manuscript was written by Noreh Rakhsh Khorshid and all authors commented on previous versions of the manuscript. All authors read and approved the final manuscript.

\section{References}

[1] H. Aktas and N. Cagman, Soft sets and soft groups, Inf. Sci. 177 (2007) 27260-2735.

[2] S. M. Anvariyeh, S. Mirvakili and B. Davvaz, On $\Gamma$-hyperideals in $\Gamma$ semihypergroups, Carpathian J. Math., 26(1) (2010) 11-23.

[3] S. M. Anvariyeh, S. Mirvakili and B. Davvaz, Pawlaks approximations in $\Gamma$-semihypergroups, Comput. Math. Appl. 60 (2010) 45- 53.

[4] R. Chinram and C. Jirojkul, On bi- $\Gamma$-ideals in $\Gamma$-semigroup, Songklanakarin J. Sci. Technol., 29(1) (2007) 231-234.

[5] S. D. Comer, Polygroups derived from cogroups, J. Algebra 89, (1984) 397-405. 
[6] S. D. Comer, Extension of polygroups by polygroups and their representations using colour schemes, Lecture notes in Meth, vol. 1004, pp. 91-103. Universal Algebra and Lattice Theory (1982).

[7] P. Corsini, Prolegomena of hypergroup theory, Aviani editore., 1993.

[8] P. Corsini and V. Leoreanu-Fotea, Applications of hyperstructure theory, Springer Science and Business Media., 5 (2013).

[9] B. Davvaz and V. Leoreanu-Fotea, Structures of fuzzy $\Gamma$-hyperideals of $\Gamma$-semihypergroups, J. Multiple-valued Logic and Soft computing., 19(2012) 519-535.

[10] B. Davvaz and V. Leoreanu-Fotea, Hyperring theory and applications, International Academic Press USA (2007).

[11] S. O. Dehkordi and B. Davvaz, A strong regular relation on $\Gamma-$ semihyperrings, J. Sci. I.R. Iran., 22(3)(2011) 257-266.

[12] S. O. Dehkordi and B. Davvaz, $\Gamma$-semihyperrings: Approximations and rough ideals, Bull. Malays. Math. Sci. Soc. (2)35 (2012) 1035-1047.

[13] S. O. Dehkordi and B. Davvaz, Г-semihyperrings: ideals, homomorphisms and regular relations, Afr. Mat., 26(5) (2015) 849-861.

[14] B. Davvaz and Mahdavipour, Roughness in modules, Inform. Sci. 176 (2006) 3658-3674.

[15] F. Feng, X. Liu, V. Leoreanu-Fotea and Y. B. Jun, Soft sets and soft rough sets, Information Sciences., 181(6) (2011) 1121-1137.

[16] F. Feng, C. Li, B. Davvaz and M.I. Ali, Soft sets combined with fuzzy sets and rough sets: a tentative approach, Soft Comput. 14 (9) (2010) 899-911.

[17] F. Feng, Y. B. Jun and X. Zhao, Soft semirings, Comput. Math. Appl., 56(10) (2008) 2621-2628.

[18] S. Ostadhadi-Dehkordi and M. Heidari, General $\Gamma$-hypergroups: $\Theta$ relation, T- Functor and Fundamental groups, Bull. Malays. Math. Sci. Soc. (2)37(3)(2014) 907-921.

[19] W.L.Gau and D.J.Buehrer, Vague sets, IEEE Trains System Man Cybernet 23(2) (1993) 610-614. 
[20] D. Heidari and B. Davvaz, $\Gamma$-hypergroups and $\Gamma$-semihypergroups associated to binary relations, Iranian Journal of Science and Technology., 35(2) (2011) 69-80.

[21] D. Heidari, S. O. Dehkordi and B. Davvaz, Г-Semihypergroups and their properties, U.P.B. Sci. Bull., Series A, 72 (2010) 197-210.

[22] K. Hila, B. Davvaz and J. Dine, Study on the structure of $\Gamma$ semihypergroups, Commun Algebra., 40(8) (2012) 2932-2948.

[23] W. Jantanan and T. Changpas, On $(m, n)$-regularity of $\Gamma$-semigroup, Thai Journal of Math., 13(1) (2015) 137-145.

[24] O. Kazanci, Sultan Yamak and B. Davvaz, The lower and upper approximations in a quotient hypermodule with respect to fuzzy sets, Inform. Sci. 178(10) (2008) 2349-2359.

[25] J. Liang, J.Wang and Y. Qian, A new measure of uncertainty based on knowledge granulation for rough sets, Inform. Sci. 179 (2009) 458-470.

[26] X. Ma, J. Zhan and B. Davvaz, Applications of rough soft sets to Krasner $(m, n)$-hyperrings and corresponding decision making, Filomat, 32(19) (2018) 6599-6614.

[27] F. Marty, Sur une generalization de la notion de group, $8^{\text {th }}$ Congress Math.Scandinaves (1934) 45-49.

[28] D. Q. Miao, Y. Zhao, Y. Y. Yao, H.X. Li and F. F. Xu, Relative reducts in and inconsistent decision tables of the Pawlak rough set model, Inform. Sci. 179 (2009) 4140-4150.

[29] P. K. Maji, A. R. Roy and R. Biswas, An application of soft sets in a decision making problem, Comput. Math. Appl., 44 (2002) 1077-1083.

[30] P. K. Maji, R. Biswas and A. R. Roy, Soft set theory, Comput. Math. Appl., 45 (2003) 555-562.

[31] Z. Meng and Z. Shi, A fast approach to attribute reduction in incomplete decision systems with tolerance relation based rough sets, Inform. Sci. 179 (2009) 2774- 2793.

[32] J. Mittas, Hypergroupes canoniques, Math. Balkanica, Beograd 2, (1972) 165-179. 
[33] D. A. Molodtsov, Soft set theory,first results, Comput. Math. Appl., 37 (1999) 19-31.

[34] D. A. Molodtsov, The description of a dependence with the help of soft sets, J. Comput. Sys. Sc. Int., 40(6) (2001) 977-984.

[35] D. A. Molodtsov, The theory of soft sets (in Russian), URSS Publishers, Moscow, (2004).

[36] C. H. Park, Y. B. Jun and M. A. Ozturk, Soft WS-algebras, Commun. Korean Math. Soc., 23(3) (2008) 313-324.

[37] Z. Pawlak, Rough sets, International Journal of Information and computer sciences 11 (1982) 341-356.

[38] Z. Pawlak and A. Skowron, Rudiment of rough sets, Inform. Sci. 177 (1) (2007) 3-27.

[39] Z. Pawlak, Theoretical Aspects of Reasoning About Data, Kluwer Academic Publishers, Boston, (1991).

[40] Z. Pawlak and A. Skowron, Rudiment of rough sets, Inform. Sci. 177 (1) (2007) 3-27.

[41] D. Pei, On definable concepts of rough set models, Inform. Sci 177 (2007) 4230- 4239 .

[42] Y. Qian, J. Liang, D. Li, H, Zhang and C. Dang, Measures for evaluating the decision performance of a decisiontable in rogh set theory, Inform. Sci 177 (2008) 181-202.

[43] M. K. Sen and N. K. Saha, On Г-semigroup, I. Bull. Cal. Math. Soc., $78,(1986)$ 180-186.

[44] W. Z. Wu and W. X. Zhang, Constructive and axiomatic approaches of fuzzy approximation operators, Inform. Sci 159 (2004) 233-254.

[45] W. Z. Wu and W. X. Zhang, Neighborhood operator systems and approximations, Inform. Sci 144 (2002) 201-217.

[46] T. Xchilleri, Completely regular $\Gamma$-semigroup, International Journal of Algebra., 4(20) (2010) 995-1002.

[47] Y. Yao, Thereway decisions with probabilistic rough sets, Inform. Sci. 180 (2010) 341-353. 
[48] L. A. Zadeh, Toward a generalized theory of uncertainly (GTU).An outline, Inform. Sci. 172 (2005) 1-40.

[49] J. Zhan, X. Zhou and D. Xiang, Rough soft n-ary semigroups based on a novel congruence relation and corresponding decision making, Journal of intelligent and fuzzy systems., 33 (2017) 693-703.

[50] J. Zhan and B. Sun, Covering-based soft fuzzy rough theory and its application to multiple criteria decision making, Comp. Appl. Math. 38, 149 (2019).

[51] J. Zhan, Q. Liu and B. Davvaz, A new rough set theory: rough soft hemirings, J. Intell. Fuzzy Systems 28 (2015) 1687-1697 